\title{
Small-gain stability theorems for positive Lur'e inclusions
}

\author{
Chris Guiver ${ }^{1}\left[\right.$ [D $\cdot$ Hartmut Logemann ${ }^{1} \cdot$ Björn Rüffer ${ }^{2}$ \\ Received: 28 June 2017 / Accepted: 2 August 2018 / Published online: 23 August 2018 \\ (c) The Author(s) 2018
}

\begin{abstract}
Stability results are presented for a class of differential and difference inclusions, so-called positive Lur'e inclusions which arise, for example, as the feedback interconnection of a linear positive system with a positive set-valued static nonlinearity. We formulate sufficient conditions in terms of weighted one-norms, reminiscent of the small-gain condition, which ensure that the zero equilibrium enjoys various global stability properties, including asymptotic and exponential stability. We also consider input-to-state stability, familiar from nonlinear control theory, in the context of forced positive Lur'e inclusions. Typical for the study of positive systems, our analysis benefits from comparison arguments and linear Lyapunov functions. The theory is illustrated with examples.
\end{abstract}

Keywords Differential inclusion · Exponential stability · Input-to-state stability · Lur'e systems · Population biology $\cdot$ Positive systems

Mathematics Subject Classification 34A60 $\cdot 37$ N35 $\cdot 39$ A30 $\cdot$ 47B65 $\cdot 93$ D20

\section{Introduction}

Positive dynamical systems, or simply positive systems, are dynamical systems where the evolution map leaves a positive cone invariant. The most natural positive cone is the nonnegative orthant of Euclidean space, equipped with the partial order of component

\footnotetext{
$\triangle$ Chris Guiver

c.guiver@bath.ac.uk

Hartmut Logemann

h.logemann@bath.ac.uk

Björn Rüffer

bjorn.ruffer@newcastle.edu.au

1 Department of Mathematical Sciences, University of Bath, Bath, UK

2 School of Mathematical and Physical Sciences, The University of Newcastle, Callaghan, NSW 2308, Australia
} 
wise inequality, noting also the often interchangeable use of the words "positive" and "nonnegative" in this context. The study of positive systems is motivated by numerous applications across a diverse range of scientific and engineering contexts, such as communications, logistics, economics, biology, chemistry, and ecology. Invariance of a positive cone captures the essential property that state-variables of positive systems, typically modelling abundances or concentrations, must take nonnegative values to be meaningful. Consequently, positive systems are well-studied objects, with textbooks which address the subject including [1-6]. The study of positive systems described by linear dynamic equations is grounded in the seminal work by Perron and Frobenius in the early 1900s on irreducible and primitive matrices. Attention has more recently turned to generalisations of the Perron-Frobenius theorem to nonlinear maps, including [7-9], for instance. Related to the theory of positive systems is the theory of monotone dynamical systems, where solutions inherit the same ordering (that is, with respect to the partial order which defines the positive cone) throughout time as their initial states; see, for example [10,11] or [12], and the references therein.

The breadth and depth of applications of positive and monotone systems has generated significant interest in their control $[1,13]$ which is currently an active research field. The analysis of positive control systems benefits from readily constructed and easily scalable Lyapunov functions [14]. In addition to numerous important examples, control of positive systems is motivated by the challenges which nonnegativity constraints place, since subtraction is not always well-defined in positive cones, for instance. As such, the following fundamental facets of linear control theory all require different treatments for positive systems: reachability and controllability $[15,16]$, observability [17], realisability [18,19] and stabilisability [20,21]. However, the additional structure afforded by positivity is often intuitive, mathematically helpful and thus simplifies matters. For example, in classical robust control, the complex and real stability radii, introduced in $[22,23]$ are, in general, different for linear systems, but are known to be equal for linear positive systems, see [24].

Differential inclusions arise as the appropriate mathematical framework for rigorously describing the solutions of differential equations specified by discontinuous functions and are now a classical subject addressed in many textbooks, including [2527]. Their study has partly been motivated by optimal control theory [28], developed in economics and engineering, see also [29,30]. Crucial to the mathematical underpinning of differential inclusions is the concept of a set-valued (or multi-valued) map [31], one of the building blocks of non-smooth analysis. In systems and control theory, differential inclusions provide a toolbox for modelling hybrid systems [32], robust control $[33,34]$ as well as hysteresis effects, such as backlash or play $[35,36]$.

We consider the class of positive differential inclusions

$$
\dot{x}-A x \in B F(C x), \quad x(0)=x^{0},
$$

so-called Lur'e inclusions, where $A, B$ and $C$ are appropriately sized matrices, with certain nonnegativity properties and $F$ is a nonnegative set-valued function. Differential inclusions of the form (1.1) often arise in closed-loop from the linear control system 


$$
\dot{x}=A x+B u, \quad x(0)=x^{0}, \quad y=C x,
$$

in feedback connection with the static set-valued (nonlinear) output feedback

$$
u \in F(y)
$$

and generalise the well-known and important class of nonlinear control systems referred to as (positive) Lur'e or Lurie systems, to which (1.1) reduces if $F$ is singletonvalued.

In the usual situation that $0 \in F(0)$, it follows that zero is an equilibrium of (1.1). We present sufficient conditions in Theorem 2.5 for the zero equilibrium to be globally stable, asymptotically stable, and exponentially stable. We note that in a dynamical systems context, exponential stability is in many respects a more important and natural notion of stability than asymptotic stability alone, see [37]. Our results are instances of so-called absolute stability theory; see, for example [36,38-42] and the references therein, in that we formulate assumptions on the transfer function $\mathbf{G}(s)=C(s I-$ $A)^{-1} B$, where $s$ is a complex-variable, which ensure the respective notions of stability for all set-valued functions $F$ for which the "product" $\mathbf{G}(0) F(y)$ satisfies certain norm bounds. The conditions may be interpreted as small-gain conditions in a weighted one-norm. In the spirit of absolute stability theory, stability of the zero equilibrium is determined by the norm-estimates and not by the individual nonlinearity $F$ itself. The inherent robustness to uncertainty in $F$ adds to the appeal and utility of absolute stability results. As corollaries we obtain stability results for certain time-varying Lur'e inclusions and to systems of positive Lur'e differential inequalities, both of which we describe. Our analysis crucially depends on the positive systems structure as we make extensive use of comparison arguments, and linear Lyapunov functions, which also go by the names of linear copositive Lyapunov functions [43] and are examples of sum-separable Lyapunov functions [44]. These techniques are not applicable in the general (non-positive) case, where, for example (see [47, Theorem 3.2]), quadratic Lyapunov functions are used which come from classical systems theoretic concepts such as the solutions of controller/observer Lyapunov equations or algebraic Riccati equations associated with the bounded real lemma or positive real lemma.

Related to (1.1) is the forced version

$$
\dot{x}-A x \in B F(C x)+D, \quad x(0)=x^{0},
$$

where $D$ is a set-valued function which models external forcing or disturbances. Clearly, when $D(t)=\{0\}$ for all $t \geq 0$, then (1.1) and (1.3) coincide. A sample forced Lur'e system is depicted in a block diagram arrangement in Fig. 1. We comment that (1.3) includes the situation wherein the forcing acts through $B$, that is, $D=B E$, for another set-valued function $E$, in which case (1.3) is the feedback interconnection of (1.2) and

$$
u \in F(y)+E .
$$

Here the natural notion of stability, now of the zero equilibrium pair ( $x=0, D=\{0\}$ ) of (1.3), is so-called input-to-state stability (ISS) initiated in [45]; see as well the 


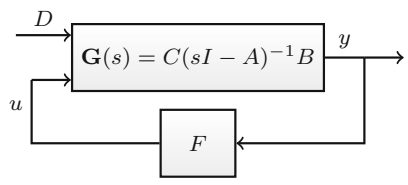

Fig. 1 Block diagram of the forced Lur'e system (1.3)

tutorial paper [46]. Roughly, ISS means that the map from $\left(x^{0}, D\right)$ to $\|x(t)\|$ has "nice" uniform boundedness properties, where $x$ is a solution of (1.3). Our Theorem 2.11 states that the same assumptions which ensure exponential stability of zero in (1.1) also ensure that the zero equilibrium pair of (1.3) enjoys the exponential ISS property, paralleling recent findings in $[47,48]$.

The approach we adopt readily admits treatment of positive Lur'e difference inclusions, that is,

$$
x^{+}-A x \in B F(C x)+D, \quad x(0)=x^{0},
$$

where $x^{+}$denotes the image of $x$ under the left-shift operator, viz. $x^{+}(t)=x(t+1)$, for all $t \in \mathbb{N}_{0}$. In Theorem 3.1 we present analogous sufficient small-gain conditions for various global stability properties of (1.4).

The motivation for the current study is to derive stability results for multivariable (that is, multi-input multi-output, or MIMO) positive Lur'e inclusions-nonlinear positive control systems - which, as already stated, arise in a variety of applied scenarios. Of particular interest to the authors are models arising in biology and ecology, naturally positive systems, which are the subject of Example 4.2. In this context, Lur'e difference equations have been proposed as models in, for example [49-52], and have also been proposed for models of plant species with seed banks [53]. Briefly, this connection has been made as Lur'e systems allow both vital or transition rates which are density-independent (that is, linear) and density-dependent (that is, nonlinear), the latter facilitating the modelling of Allee [54-56], competition or crowding effects. As inherently "noisy" systems, not described by classical, well-understood equations of motion, there is often much uncertainty in parametrising ecological models, with different qualitative trends being observed in different parametrisations [57]. The robustness to such uncertainty afforded by the treatment of dynamic inclusions and set-valued analysis is thus especially applicable and appealing. Although clearly existing results for the absolute stability of Lur'e inclusions such as those in $[36,48]$ do apply here, since these papers are not aimed at positive systems, their results are necessarily more conservative.

There is some partial overlap between the present work and [58,59], where absolute stability results for continuous- and discrete-time positive dynamical systems (of differential or difference equations, respectively, including Lur'e systems) are presented, although for unforced systems only. These results also appear in the monograph [1, Chap. 5] by the same authors. In [1,58,59] a linear dissipativity theory approach is adopted and we compare results in Remarks 2.14 and 3.4. Our work in part extends that of $[49,60]$ which consider positive Lur'e difference and differential equations, respectively, both with scalar nonlinearities. It is possible in these specific situations to present "trichotomies of stability" explicitly in terms of the model data, which ensure 
that, under certain assumptions, solutions either converge to the zero equilibrium, or a unique non-zero equilibrium, or diverge. Similar limit set trichotomies have been established for various classes of monotone discrete-time dynamical systems in [2,61, Chap. 6] for finite-dimensional systems and in [12,50,51] for infinite-dimensional systems. In Remark 3.5, we compare some of our findings with those from the monotone systems and control literature, namely [62,63]. In the present paper we restrict attention to stability of the zero equilibrium alone and do not consider limit set trichotomies.

The paper is organised as follows. Sections 2 and 3 contain our main results, namely, global stability properties of the Lur'e differential inclusions (1.1) and (1.3), and the Lur'e difference inclusion (1.4), respectively. Section 4 contains three examples discussed in detail. We present some brief summarising remarks in Sect. 5.

Notation We collect notation and terminology used in the sequel. The symbols $\mathbb{N}$ and $\mathbb{R}$ denote the sets of positive integers and real numbers, respectively, and $\mathbb{N}_{0}=\{0\} \cup \mathbb{N}$. For $n, m \in \mathbb{N}$, we let $\underline{n}:=\{1,2, \ldots, n\}, \mathbb{R}^{n}$ and $\mathbb{R}^{n \times m}$ denote usual $n$-dimensional Euclidean space, and the set of $n \times m$ matrices with real entries, respectively. The superscript ${ }^{T}$ denotes both matrix and vector transposition. For $M, N \in \mathbb{R}^{n \times m}$ with entries $m_{i j}$ and $n_{i j}$, respectively, we write

$$
\begin{array}{ll}
M \leq N & \text { if } m_{i j} \leq n_{i j} \text { for all } i \in \underline{n}, j \in \underline{m}, \\
M<N & \text { if } M \leq N \text { and } M \neq N, \\
M \ll N & \text { if } m_{i j}<n_{i j} \text { for all } i \in \underline{n}, j \in \underline{m},
\end{array}
$$

with the corresponding respective conventions for $\geq,>$ and $\gg$. If $M \in \mathbb{R}_{+}^{n \times m}$ or, equivalently, $0 \leq M$, then we say that $M$ is nonnegative. We call $M$ positive or strictly positive if $0<M$ or $0 \ll M$, respectively, noting that there are different conventions present in the literature for the term positive matrix. Given $v \in \mathbb{R}_{+}^{n}, 0 \ll v$, we let

$$
\underset{v}{v}:=\min _{j \in \underline{n}} v_{j}>0 \quad \text { and } \quad|x|_{v}:=v^{T}|x|=\sum_{j=1}^{n} v_{j}\left|x_{j}\right|, \quad \forall x=\left(x_{1} \ldots x_{n}\right)^{T} \in \mathbb{R}^{n}
$$

where the $i$-th component of $|x| \in \mathbb{R}_{+}^{n}$ is defined to be $\left|x_{i}\right|$. We note that $|\cdot|_{v}$ is a norm on $\mathbb{R}^{n}$ and, if $x \in \mathbb{R}_{+}^{n}$, then $|x|_{v}=v^{T} x$.

We recall that a nonnegative square matrix $M \in \mathbb{R}_{+}^{n \times n}$ is irreducible if, and only if, for each $i, j \in \underline{n}$ there exists $k \in \mathbb{N}$ such that the $(i, j)$-th entry of $M^{k}$ is positive. Strictly positive matrices are evidently irreducible, and small irreducible matrices (which are not strictly positive) include

$$
\left(\begin{array}{ll}
0 & 1 \\
1 & 0
\end{array}\right) \text { and }\left(\begin{array}{lll}
0 & 0 & 1 \\
1 & 0 & 0 \\
0 & 1 & 0
\end{array}\right)
$$

Irreducible matrices presently play a role because Perron-Frobenius theory applies to them, which we discuss later in the text.

A matrix $M \in \mathbb{R}^{n \times n}$ is called Metzler, also known as quasi-positive or essentially nonnegative, if every off-diagonal entry is nonnegative (see, for example 
[5, Chap. 6]). We let $r(M)$ and $\alpha(M)$ denote the spectral radius and spectral abscissa of $M$, respectively, which we recall are given by

$$
r(M):=\max \{|\lambda|: \lambda \in \sigma(M)\} \quad \text { and } \alpha(M):=\max \{\operatorname{Re} \lambda: \lambda \in \sigma(M)\},
$$

where $\sigma(M)$ denotes the spectrum of $M$. For $v \in \mathbb{R}^{n},\|v\|$ and $\|M\|$ denote a (any) monotonic norm of $v$ and the corresponding induced operator norm of $M$, respectively. Recall that a norm is monotonic if for all $x, y \in \mathbb{R}^{n}$

$$
|x| \leq|y| \Rightarrow\|x\| \leq\|y\| \text { where }|x|:=\left(\left|x_{1}\right| \ldots\left|x_{n}\right|\right)^{T}
$$

Every $p$-norm for $1 \leq p \leq \infty$ is monotonic. We let $\|\cdot\|_{1}$ denote both the one-norm and induced one-norm.

For $v, w \in \mathbb{R}^{n}$ with $v \leq w$, we define the order interval $[v, w] \subseteq \mathbb{R}^{n}$ as

$$
[v, w]:=\left\{x \in \mathbb{R}^{n}: v \leq x \leq w\right\}
$$

which, in light of the componentwise definition of the partial order $\leq$, is equal to the Cartesian product of intervals, viz.

$$
\begin{aligned}
{[v, w] } & =\left[v_{1}, w_{1}\right] \times\left[v_{2}, w_{2}\right] \times \ldots \times\left[v_{n}, w_{n}\right] \text { where } \\
v & =\left(v_{1} \ldots v_{n}\right)^{T}, w=\left(w_{1} \ldots w_{n}\right)^{T} .
\end{aligned}
$$

The symbols $I$ and $\mathbb{1}$ denote the identity matrix and vector with every component equal to one, respectively, the size of which shall be consistent with the context. We note that with the above definitions $\|x\|_{1}=|x|_{\mathbb{1}}$, for $x \in \mathbb{R}^{n}$.

For a set $X$, we let $P(X)$ denote the power set of $X, P_{0}(X)=P(X) \backslash\{\emptyset\}$ and, if $X$ is a subset of a normed-space,

$$
\|X\|:=\sup _{x \in X}\|x\|
$$

with the convention that $\|\emptyset\| \|=0$. If $X, Y \subseteq V$ are subsets of a vector space and $T: V \rightarrow V$ a linear operator, then the sum $X+Y$ and image $T X$ are defined as

$$
X+Y:=\{x+y: x \in X, y \in Y\} \text { and } T X:=\{T x: x \in X\} .
$$

Finally, a function $g: \mathbb{R}^{n} \rightarrow \mathbb{R}^{m}$ is said to be lower semi-continuous if every component $g_{i}: \mathbb{R}^{n} \rightarrow \mathbb{R}$ is lower semi-continuous, where $i \in \underline{m}$, that is

$$
g_{i}(x) \leq \liminf _{y \rightarrow x} g_{i}(y) \quad \forall x \in \mathbb{R}^{n}
$$




\section{Continuous-time systems}

In this section we consider continuous-time Lur'e inclusions, treating the unforced and forced cases in separate subsections. We first collect some terminology and results which are used in both subsections.

Given a set-valued map $H: \mathbb{R}_{+} \times \mathbb{R}^{n} \rightarrow P_{0}\left(\mathbb{R}^{n}\right)$ and $x^{0} \in \mathbb{R}^{n}$, we say that an absolutely continuous function $x:[0, \omega) \rightarrow \mathbb{R}^{n}$ for $0<\omega \leq \infty$, is a solution of the initial value problem

$$
\dot{x}(t) \in H(t, x(t)), \quad x(0)=x^{0},
$$

if (2.1) holds for almost all $t \in[0, \omega)$. If $\omega=\infty$, then $x$ is said to be a global solution. If $x:[0, \omega) \rightarrow \mathbb{R}^{n}$ is a solution of (2.1) for some $0<\omega \leq \infty$, then $\dot{x}$ is a locally integrable selection of $t \mapsto H(t, x(t))$. Therefore, for all $0 \leq t_{1} \leq t_{2}<\omega$, the set

$$
\begin{aligned}
& \int_{t_{1}}^{t_{2}} H(s, x(s)) d s \\
& \quad:=\left\{\int_{t_{1}}^{t_{2}} h(s) d s \mid h \in L^{1}\left(\left[t_{1}, t_{2}\right] ; \mathbb{R}^{n}\right) \text { is a selection of } t \mapsto H(t, x(t))\right\},
\end{aligned}
$$

is non-empty and, with this notation, $x$ satisfies the integral inclusion

$$
x\left(t_{2}\right)-x\left(t_{1}\right) \in \int_{t_{1}}^{t_{2}} H(s, x(s)) d s
$$

We shall consider the special case of (2.1) with

$$
H(t, x)=A x+B G(t, C x)+D(t),
$$

and

$$
(A, B, C) \in \mathbb{R}^{n \times n} \times \mathbb{R}^{n \times m} \times \mathbb{R}^{p \times n}, \quad D: \mathbb{R}_{+} \rightarrow P_{0}\left(\mathbb{R}^{n}\right), \quad \text { and } G: \mathbb{R}_{+} \times \mathbb{R}^{p} \rightarrow P_{0}\left(\mathbb{R}^{m}\right),
$$

for some $m, n, p \in \mathbb{N}$. We will impose positivity and stability properties on the data in (2.4) later in the section. Combined, (2.1), (2.3) and (2.4) give rise to the system of Lur'e differential inclusions

$$
\dot{x}(t)-A x(t) \in B G(t, C x(t))+D(t), \quad x(0)=x^{0}, \quad t \in \mathbb{R}_{+} .
$$

Our primary, but not exclusive, focus is the autonomous case wherein $G(t, y)=F(y)$, for some $F: \mathbb{R}^{p} \rightarrow P_{0}\left(\mathbb{R}^{n}\right)$, so that (2.5) reduces to (1.3). The set-valued forcing term $D: \mathbb{R}_{+} \rightarrow P_{0}\left(\mathbb{R}^{n}\right)$ does not play a role in Sect. 2.1, where (1.1) is considered by taking $D=\{0\}$. As mentioned in the Introduction, when $G$ is singleton-valued then (2.5) simplifies to a system of Lur'e differential equations; a special case which we consider in Sect. 2.3. We next state and prove a "variation of parameters" expression for solutions of forced Lur'e inclusions. No stability or positivity properties are required for this result. 
Lemma 2.1 For model data (2.4) and $x^{0} \in \mathbb{R}^{n}$, let $x:[0, \omega) \rightarrow \mathbb{R}^{n}$ denote a solution of (2.5) for some $\omega>0$. Then $x$ satisfies the inclusion

$$
x(t)-\mathrm{e}^{A t} x^{0} \in \int_{0}^{t} \mathrm{e}^{A(t-\tau)}[B G(\tau, C x(\tau))+D(\tau)] d \tau \quad \forall t \in[0, \omega) .
$$

Proof Let $0<t<\omega$ be fixed, but arbitrary, and define an absolutely continuous function $z$ on $[0, t]$ by $z(\tau):=\mathrm{e}^{A(t-\tau)} x(\tau)$. Obviously, $z(0)=\mathrm{e}^{A t} x^{0}$ and $z(t)=x(t)$, and a routine calculation shows that $z$ satisfies the differential inclusion

$$
\frac{\mathrm{d}}{\mathrm{d} \tau} z(\tau) \in \mathrm{e}^{A(t-\tau)}[B G(\tau, C x(\tau))+D(\tau)] \text { almost all } \tau \in(0, t) .
$$

Therefore, in light of (2.2),

$$
z(t)-z(0)=\int_{0}^{t} \frac{\mathrm{d}}{\mathrm{d} \tau} z(\tau) d \tau \in \int_{0}^{t} \mathrm{e}^{A(t-\tau)}[B G(\tau, C x(\tau))+D(\tau)] d \tau,
$$

which implies that

$$
x(t)-\mathrm{e}^{A t} x^{0} \in \int_{0}^{t} \mathrm{e}^{A(t-\tau)}[B G(\tau, C x(\tau))+D(\tau)] d \tau .
$$

Since $0<t<\omega$ was arbitrary, we conclude that (2.6) holds.

The following equivalence, known as "loop-shifting" in the control engineering jargon, shall also play a key role, and is easily established. Namely, for fixed $K \in$ $\mathbb{R}^{m \times p}, x^{0} \in \mathbb{R}^{n}$ and model data (2.4), the function $x$ is a solution of (2.5) if, and only if, $x$ is a solution of

$$
\dot{x}(t)-(A+B K C) x(t) \in B(G(t, C x(t))-K C x(t))+D(t), \quad x(0)=x^{0}, \quad t \in \mathbb{R}_{+} .
$$

We introduce the positivity and stability properties of $A, B, C, D$ and $G$ as in (2.4):

$$
\begin{aligned}
& \text { (A1) }(A, B, C) \in \mathbb{R}^{n \times n} \times \mathbb{R}_{+}^{n \times m} \times \mathbb{R}_{+}^{p \times n}, A \text { is Metzler, } D: \mathbb{R}_{+} \rightarrow P_{0}\left(\mathbb{R}_{+}^{n}\right) \text { and } \\
& G: \mathbb{R}_{+} \times \mathbb{R}_{+}^{p} \rightarrow P_{0}\left(\mathbb{R}_{+}^{m}\right) \text {; }
\end{aligned}
$$

(A2) $\alpha(A)<0$;

(A3) there exists $R>0$ such that

$$
\|w\| \leq R\|y\| \quad \forall w \in G(t, y) \quad \forall t \in \mathbb{R}_{+} \forall y \in \mathbb{R}_{+}^{p} .
$$

We comment that we shall interpret (A1) and (A3) in the autonomous case as well, meaning that $G(t, y)=F(y)$ for some $F: \mathbb{R}^{p} \rightarrow P_{0}\left(\mathbb{R}^{m}\right)$.

We briefly discuss an issue pertaining to domains which often arises when studying positive systems, to reconcile the general case (2.4) to the assumptions made in (A1). Indeed, under (A1), the function $G$ is only defined on $\mathbb{R}_{+} \times \mathbb{R}_{+}^{p}$. Some existence theory (see, for example [25, Theorem 1, p. 97]) for (2.1) assumes that $H: I \times \Omega \rightarrow P_{0}(X)$ 
where $I \subseteq \mathbb{R}$ and $\Omega \subseteq X \subseteq \mathbb{R}^{n}$ are open, which clearly fails with $I=\mathbb{R}_{+}$and $\Omega=\mathbb{R}_{+}^{n}$. Therefore, if $J: \mathbb{R}_{+} \times \mathbb{R}_{+}^{n} \rightarrow P_{0}\left(\mathbb{R}_{+}^{n}\right)$ denotes the right hand side of the differential inclusion (2.5) under (A1), then we extend $J$ to $\mathbb{R} \times \mathbb{R}^{n}$, and denote the extension $J_{\mathrm{e}}: \mathbb{R} \times \mathbb{R}^{n} \rightarrow P_{0}\left(\mathbb{R}_{+}^{n}\right)$, by setting $J_{\mathrm{e}}(t, x):=J(\max \{0, t\}, \mu(x))$ with $\mu: \mathbb{R}^{n} \rightarrow \mathbb{R}_{+}^{n}$ defined by

$$
\mu(x):=\left(\max \left\{0, x_{1}\right\}, \max \left\{0, x_{2}\right\} \ldots \max \left\{0, x_{n}\right\}\right)^{T} \quad \forall x=\left(x_{1} \ldots x_{n}\right)^{T} \in \mathbb{R}^{n} .
$$

Since $J$ and $J_{\mathrm{e}}$ coincide on $\mathbb{R}_{+}^{n}$, we may seek solutions of $\dot{x} \in J_{\mathrm{e}}(x)$, as an immediate consequence of the positivity assumptions in (A1) is the following.

Lemma 2.2 Under assumption (A1), every solution $x:[0, \omega) \rightarrow \mathbb{R}^{n}$ of (2.5) with $x^{0} \in \mathbb{R}_{+}^{n}$ takes values in $\mathbb{R}_{+}^{n}$ for all $t \in[0, \omega)$.

Proof The claim follows from the variation of parameters inclusion (2.6) combined with the nonnegativity assumptions in (A1). Crucially, we have used that $A$ is Metzler if, and only if, $\mathrm{e}^{A t}>0$ for all $t \geq 0$; see, for example [10, Section 3.1].

We conclude this section by commenting that the loop-shifting property, see (2.7), holds without the assumptions (A1)-(A3). It demonstrates that we may replace $A$ and $G$ in (1.3) by $A+B K C$ and $(t, y) \mapsto G(t, y)-K y$, respectively. In particular, $A$ may not satisfy assumption (A2), but $A+B K C$ may do so, for some $K \in \mathbb{R}^{p \times m}$. In other words, this means that there is a so-called stabilising static output feedback $u=K y$ for the linear system (1.2), which is the approach we take in Example 4.1. Of course, in the current setting of positive Lur'e inclusions, the choice of $K$ shall be constrained by the requirement that $A+B K C$ and $(t, y) \mapsto G(t, y)-K y$ satisfy (A1) as well, which may be infeasible in some cases.

\subsection{Unforced systems}

We consider the Lur'e inclusion (1.1) with assumptions (A1)-(A3). Recall from the previous section that this is a special case of (2.5). We note that the assumptions on $A, B, C$ and $F$ do not a priori guarantee that (1.1) admits solutions. Existence of solutions is not the focus of the present investigation, primarily as it is an extensively studied subject in the literature. That said, we do make some comments and provide some references regarding the existence of solutions. Recall the extension $J_{\mathrm{e}}$ from Sect. 2, defined in terms of $\mu$ from (2.8). The following lemma shows that $J_{\mathrm{e}}$ inherits many useful properties from $J$. The proofs are readily established once it is noted that $\mu$ is Lipschitz with Lipschitz constant equal to one.

Lemma 2.3 Let $J: \mathbb{R}_{+} \times \mathbb{R}_{+}^{n} \rightarrow P_{0}\left(\mathbb{R}_{+}^{n}\right)$ and define $J_{\mathrm{e}}(t, x):=J(\max \{0, t\}, \mu(x))$ where $\mu$ is given by (2.8). If $J$ has any of the following properties: bounded, closed valued, convex valued, upper semi-continuous, lower semi-continuous, then $J_{\mathrm{e}}$ has the corresponding property.

Examples of results ensuring existence of solutions of (1.1) include [25, Theorem 3, p. 98], [26, Proposition 6.1, p. 53] and [27, Theorem 7.5.1, p. 279]. The 
results [25, Theorems 1 and 4, p. 97, p. 101], [26, Lemma 5.1, p. 53] and [26, Theorem 6.1, p. 53] provide conditions under which global solutions of (1.1) exist, from which we obtain the following.

Proposition 2.4 Given the Lur'e inclusion (1.1), assume that (A1) and (A3) hold and that $F$ is upper semi-continuous with closed, convex values. Then, for all $x^{0} \in \mathbb{R}_{+}^{n}$, there is a global solution of (1.1), and every solution may be extended to a global solution. Further, every solution $x$ satisfies $x(t) \in \mathbb{R}_{+}^{n}$ for all $t$ where $x(t)$ is defined.

Proof The lemma follows from applications of [26, Lemma 5.1, p. 53] and [26, Corollary 5.2, p. 58], which establish existence of solutions, and extension to global solutions, respectively. Lemma 2.2 ensures that every solution is nonnegative.

We shall later briefly consider non-autonomous versions of (1.1), specifically (2.5). In this context [26, Theorem 5.2, p. 58] provides further assumptions on $G$ which, combined with (A1)-(A3), guarantee the existence of global solutions. In fact, under (A1)-(A3), any result guaranteeing existence of local solutions which uses continuous (or Caratheodory) selections of $x \mapsto A x+B F(C x)$ (or $(t, x) \mapsto$ $A x+B G(t, C x))$ will, in fact, ensure existence of global solutions by well-known theory of maximally defined solutions of ordinary differential equations (see the proof of [25, Theorem 1, p. 97]).

The main result of this section is presented next, and contains a suite of stability results for (1.1) formulated in terms of weighted one-norm inequalities and $\mathbf{G}(0)$, assuming that global solutions exist. Here $\mathbf{G}$ denotes the transfer function of the triple $(A, B, C)$, that is, $\mathbf{G}(s)=C(s I-A)^{-1} B$, where $s$ is a complex variable. Recall that a matrix $A \in \mathbb{R}^{n \times n}$ is Metzler with $\alpha(A)<0$ if, and only if, $-A^{-1}>0$ (see, for example [5, Characterisation $\mathrm{N}_{38}$ in Section 6.2] or [66, characterisation $\left.\mathrm{F}_{15}\right]$ ). Consequently, under assumptions (A1) and (A2), it follows that $\mathbf{G}(0)=-C A^{-1} B \in \mathbb{R}_{+}^{p \times m}$.

Theorem 2.5 Given the Lur'e inclusion (1.1), assume that (A1)-(A3) hold.

(i) If there exists a strictly positive $v \in \mathbb{R}_{+}^{p}$ such that

$$
|\mathbf{G}(0) w|_{v} \leq|y|_{v} \quad \forall w \in F(y) \quad \forall y \in \mathbb{R}_{+}^{p},
$$

then there exists $\Gamma>0$ such that, for all $x^{0} \in \mathbb{R}_{+}^{n}$, every global solution $x$ of (1.1) satisfies

$$
\|x(t)\| \leq \Gamma\left\|x^{0}\right\| \quad \forall t \in \mathbb{R}_{+} .
$$

(ii) If there exist a strictly positive $v \in \mathbb{R}_{+}^{p}$ and a lower semi-continuous function $e: \mathbb{R}_{+}^{p} \rightarrow \mathbb{R}_{+}$such that

$e(y)>0$ and $|\mathbf{G}(0) w|_{v}+e(y) \leq|y|_{v} \quad \forall w \in F(y) \quad \forall y \in \mathbb{R}_{+}^{p} \backslash\{0\}$, (2.10)

then, for all $x^{0} \in \mathbb{R}_{+}^{n}$, every global solution $x$ of (1.1) satisfies $x(t) \rightarrow 0$ as $t \rightarrow \infty$. 
(iii) If there exist a strictly positive $v \in \mathbb{R}_{+}^{p}$ and $\rho \in(0,1)$ such that

$$
|\mathbf{G}(0) w|_{v} \leq \rho|y|_{v} \quad \forall w \in F(y) \quad \forall y \in \mathbb{R}_{+}^{p},
$$

then there exist $\Gamma, \gamma>0$ such that, for all $x^{0} \in \mathbb{R}_{+}^{n}$, every global solution $x$ of (1.1) satisfies

$$
\|x(t)\| \leq \Gamma \mathrm{e}^{-\gamma t}\left\|x^{0}\right\| \quad \forall t \in \mathbb{R}_{+} .
$$

The notion of stability concluded in statement (i) is often called "stability in the large", see [36, Definition 3], which, when combined with statements (ii) and (iii) yield that the zero equilibrium of (1.1) is globally asymptotically and globally exponentially stable, respectively. Before proving the above theorem, we provide some commentary on assumptions (2.9)-(2.11).

Remark 2.6 The weighted one-norm estimates (2.9)-(2.11) provide conditions on the norm of the "product" $\mathbf{G}(0) F(y)$ and not on a product of norms. Sufficient conditions for (2.9)-(2.11) are linear constraints which are reminiscent of sector-type conditions in a nonnegative orthant. Namely, if there exists an irreducible matrix $M \in \mathbb{R}_{+}^{p \times p}$ with $r(M) \leq 1$ such that

$$
\mathbf{G}(0) w \leq M y \quad \forall w \in F(y) \quad \forall y \in \mathbb{R}_{+}^{p},
$$

then (2.9) holds. To see this, multiply both sides of (2.12) on the left by $v^{T}$, a strictly positive left eigenvector of $M$ corresponding to the eigenvalue $r(M)$, the existence of which is ensured by the Perron-Frobenius Theorem (see, for example [5, Theorem 1.4,p. 27]). Further, if there exists an irreducible matrix $M \in \mathbb{R}_{+}^{p \times p}$ with $r(M) \leq 1$ and a lower semi-continuous function $\zeta: \mathbb{R}_{+}^{p} \rightarrow \mathbb{R}_{+}^{p}$ such that

$$
\zeta(y)>0 \text { and } \mathbf{G}(0) w+\zeta(y)<M y \quad \forall w \in F(y) \quad \forall y \in \mathbb{R}_{+}^{p} \backslash\{0\}
$$

then (2.10) holds with $e:=v^{T} \zeta$, by the same argument as above. If there exists a nonnegative (not necessarily irreducible) matrix $M$ which satisfies (2.12) with the property that $r(M)<1$, then (2.11) holds.

Proof of Theorem 2.5 Throughout the proof, we let $x: \mathbb{R}_{+} \rightarrow \mathbb{R}_{+}^{n}$ denote a global solution of (1.1) for given $x^{0} \in \mathbb{R}_{+}^{n}$.

(i): As $\mathbf{G}(0) \geq 0$, the condition (2.9) may be rewritten as

$$
v^{T} \mathbf{G}(0) F(C x(t)) \subseteq\left[0, v^{T} C x(t)\right] \quad \forall t \in \mathbb{R}_{+} .
$$

Multiplying both sides of (1.1) by $-v^{T} C A^{-1}$ and invoking (2.13) yields that

$$
\begin{aligned}
-v^{T} C A^{-1} \dot{x}(t) & \in-v^{T} C A^{-1}[A x(t)+B F(C x(t))]=-v^{T} C x(t)+v^{T} \mathbf{G}(0) F(C x(t)) \\
& \subseteq\left[-v^{T} C x(t), 0\right] \text { for almost all } t \geq 0
\end{aligned}
$$


whence

$$
-v^{T} C A^{-1} \dot{x}(t) \leq 0 \text { for almost all } t \geq 0,
$$

and, further,

$$
0 \leq-v^{T} C A^{-1} x(t) \leq-v^{T} C^{-1} A x^{0} \quad \forall t \in \mathbb{R}_{+} .
$$

We claim that there exists $\theta>0$ such that

$$
\theta z \leq-A^{-1} z \quad \forall z \in \mathbb{R}_{+}^{n}
$$

To that end, choose $\beta>0$ such that $A+\beta I \geq 0$, so that in particular

$$
\mathrm{e}^{(A+\beta I) t}=\sum_{k=0}^{\infty} \frac{t^{k}}{k !}(A+\beta I)^{k} \geq I \quad \forall t \in \mathbb{R}_{+}
$$

As $A$ is Metzler with $\alpha(A)<0$, in light of (2.17) we have, for $z \in \mathbb{R}_{+}^{n}$,

$$
-A^{-1} z=\int_{0}^{\infty} \mathrm{e}^{A t} z d t=\int_{0}^{\infty} \mathrm{e}^{(A+\beta I) t} \mathrm{e}^{-\beta t} z d t \geq \int_{0}^{\infty} \mathrm{e}^{-\beta t} z d t=\frac{z}{\beta},
$$

which is (2.16) with $\theta:=1 / \beta>0$. Using (2.15) and (2.16) shows that

$$
\theta \underset{\sim}{v}\|C x(t)\|_{1} \leq \theta v^{T} C x(t) \leq-v^{T} C A^{-1} x(t) \leq-v^{T} C A^{-1} x^{0} \quad \forall t \in \mathbb{R}_{+},
$$

which implies, by norm equivalence, that there exists $P>0$ such that

$$
\|C x(t)\| \leq P\left\|x^{0}\right\| \quad \forall t \in \mathbb{R}_{+} .
$$

Invoking Lemma 2.1, the variation of parameters inclusion (2.6) with $G(t, C x(t))=$ $F(C x(t))$ and $D=\{0\}$, combined with (A3) and (2.18), we may estimate

$$
\|x(t)\| \leq\left(\left\|\mathrm{e}^{A t}\right\|+R P \int_{0}^{t}\left\|\mathrm{e}^{A(t-\tau)} B\right\| d \tau\right)\left\|x^{0}\right\| \quad \forall t \in \mathbb{R}_{+},
$$

whence, by (A2), we conclude that statement (i) holds.

(ii): Our hypotheses ensure that statement (i) holds. First, suppose that

$$
C x(t) \rightarrow 0 \text { as } t \rightarrow \infty
$$

and fix $\varepsilon>0$. We note that for any $T_{1} \geq 0$, we may use (A2), (A3) and (2.19) to estimate that $\xi$ satisfying

$$
\xi(t) \in \int_{T_{1}}^{t} \mathrm{e}^{A(t-\tau)} B F(C x(\tau)) d \tau \quad \forall t \geq T_{1},
$$


admits the estimate

$$
\begin{aligned}
\|\xi(t)\| & \leq \int_{T_{1}}^{t}\left\|\mathrm{e}^{A(t-\tau)}\right\|\|B\|\|F(C x(\tau))\| d \tau \leq\|B\| R \int_{T_{1}}^{t}\left\|\mathrm{e}^{A(t-\tau)}\right\|\|C x(\tau)\| d \tau \\
& \leq \frac{\varepsilon}{2}
\end{aligned}
$$

for $T_{1}>0$ sufficiently large. Consequently, $\|\xi(t)\| \leq \varepsilon / 2$ for all $t \geq T_{1}$. Since $x$ satisfies

$$
x(t)-\mathrm{e}^{A\left(t-T_{1}\right)} x\left(T_{1}\right) \in \int_{T_{1}}^{t} \mathrm{e}^{A(t-\tau)} B F(C x(\tau)) d \tau \quad \forall t \geq T_{1},
$$

(which follows easily from (2.6)), invoking (A2) gives $T_{2}>T_{1}$ such that for all $t \geq T_{2}$

$$
\left\|x(t)-\mathrm{e}^{A\left(t-T_{1}\right)} x\left(T_{1}\right)\right\| \leq \frac{\varepsilon}{2} \Rightarrow\|x(t)\| \leq\left\|\mathrm{e}^{A\left(t-T_{1}\right)} x\left(T_{1}\right)\right\|+\frac{\varepsilon}{2}<\varepsilon
$$

hence $\lim _{t \rightarrow \infty} x(t)=0$ as $t \rightarrow \infty$, as required, provided that (2.19) holds.

Therefore, it remains to establish (2.19). For which purpose, fix $x^{0} \in \mathbb{R}_{+}^{n} \backslash\{0\}$ and, seeking a contradiction, suppose that (2.19) fails. Then there exist a sequence $\left(t_{k}\right)_{k \in \mathbb{N}} \subseteq \mathbb{R}_{+}$and $\varepsilon>0$ such that $t_{k} \nearrow \infty$ as $k \rightarrow \infty$ and

$$
2 \varepsilon \leq\left\|C x\left(t_{k}\right)\right\| \quad \forall k \in \mathbb{N} .
$$

By statement (i) we have that $x$ is bounded, and hence from (1.1) and (A3) it follows that $\dot{x}$ is bounded as well. Hence $x$ is uniformly continuous, and so is $C x$. Consequently, there exists $\delta>0$ such that

$$
\varepsilon \leq\|C x(t)\| \leq \Gamma\|C\|\left\|x^{0}\right\| \quad \forall t \in\left[t_{k}, t_{k}+\delta\right] \quad \forall k \in \mathbb{N}
$$

where we have used the bound for $x$ from statement (i). As $t_{k} \nearrow \infty$ as $k \rightarrow \infty$ we may assume that $t_{k+1}>t_{k}+\delta$ for all $k \in \mathbb{N}$ (by redefining the sequence $\left(t_{k}\right)_{k \in \mathbb{N}}$ if necessary). Define

$$
\mathcal{M}:=\left\{\xi \in \mathbb{R}_{+}^{p}: \varepsilon \leq\|\xi\| \leq \Gamma\|C\|\left\|x^{0}\right\|\right\}
$$

a compact set which does not contain zero. We claim that there exists $\eta>0$ such that

$$
\inf _{\substack{\xi \in \mathcal{M} \\ w \in F(\xi)}}\left[v^{T} \xi-v^{T} \mathbf{G}(0) w\right] \geq \eta
$$

To establish (2.22), note that by (2.10),

$$
\left[v^{T} \xi-v^{T} \mathbf{G}(0) w\right] \geq e(\xi) \geq \inf _{\zeta \in \mathcal{M}} e(\zeta)=: \eta>0, \quad \forall w \in F(\xi), \forall \xi \in \mathcal{M}
$$


as $e$ is a lower semi-continuous function which is positive-valued for positive arguments, and $\mathcal{M}$ is a compact set which does not contain zero.

Consider next the real-valued, nonnegative function

$$
f: \mathbb{R}_{+} \rightarrow \mathbb{R}_{+}, \quad f(t):=-v^{T} C A^{-1} x(t),
$$

which is absolutely continuous, non-increasing by (2.14) and, furthermore, satisfies

$$
\dot{f}(t)=-v^{T} C A^{-1} \dot{x}(t) \in-v^{T} C x(t)+v^{T} \mathbf{G}(0) F(C x(t)) \quad \text { for almost all } t \in \mathbb{R}_{+} .
$$

In light of (2.20), (2.22) and (2.23), we see that for every $k \in \mathbb{N}$

$$
\dot{f}(t) \in(-\infty,-\eta] \text { for almost all } t \in\left[t_{k}, t_{k}+\delta\right],
$$

which yields that

$$
f\left(t_{k}+\delta\right)-f\left(t_{k}\right) \leq-\eta \delta
$$

Since $f$ is non-increasing and $t_{k+1} \geq t_{k}+\delta$ for all $k \in \mathbb{N}$

$$
f\left(t_{k+1}\right)-f\left(t_{k}\right) \leq-\eta \delta, \quad \forall k \in \mathbb{N},
$$

showing that

$$
f\left(t_{N+1}\right)-f\left(t_{1}\right)=\sum_{k=1}^{N}\left[f\left(t_{k+1}\right)-f\left(t_{k}\right)\right] \leq-\eta \delta N \rightarrow-\infty \quad \text { as } N \rightarrow \infty
$$

which contradicts the nonnegativity of $f$.

(iii): Let $0<c_{1} \leq c_{2}$ be such that

$$
c_{1}\|y\| \leq|y|_{v} \leq c_{2}\|y\| \quad \forall y \in \mathbb{R}^{p}
$$

Fix $\varepsilon>0$ such that $\rho+\varepsilon<1$. Since $\alpha(A)<0$ and as $\mathbf{G}$ is continuous at 0 , there exists $\delta>0$ such that

$$
0<\gamma<\delta \Rightarrow \alpha(A+\gamma I)<0 \text { and }\|\mathbf{G}(-\gamma)-\mathbf{G}(0)\|<\frac{\varepsilon c_{1}}{c_{2} R}
$$

Thus, for fixed $\gamma \in(0, \delta)$, we note that $\mathbf{G}(-\gamma) \geq 0$ and, further, for $y \in \mathbb{R}_{+}^{p}$ and $w \in F(y)$,

$$
\begin{aligned}
|\mathbf{G}(-\gamma) w|_{v} & =v^{T} \mathbf{G}(-\gamma) w=v^{T}[\mathbf{G}(-\gamma)-\mathbf{G}(0)] w+|\mathbf{G}(0) w|_{v} \\
& \leq|[\mathbf{G}(-\gamma)-\mathbf{G}(0)] w|_{v}+|\mathbf{G}(0) w|_{v} \\
& \leq c_{2}\|\mathbf{G}(-\gamma)-\mathbf{G}(0)\| \cdot\|w\|+\rho|y|_{v} \leq \frac{\varepsilon c_{1}}{R}\|w\|+\rho|y|_{v} \leq(\varepsilon+\rho)|y|_{v} \\
& \leq|y|_{v},
\end{aligned}
$$


where we have used (A3), (2.11), (2.24) and (2.25). Multiplying both sides of (2.26) by $\mathrm{e}^{\gamma t}$, and taking $w \in F\left(\mathrm{e}^{-\gamma t} \xi\right)$ with $\xi \in \mathbb{R}_{+}^{p}$, we see that

$$
\left|\mathrm{e}^{\gamma t} \mathbf{G}(-\gamma) w\right|_{v} \leq \mathrm{e}^{\gamma t} \cdot\left|\mathrm{e}^{-\gamma t} \xi\right|_{v}=|\xi|_{v} \quad \forall w \in F\left(\mathrm{e}^{-\gamma t} \xi\right), \quad \forall \xi \in \mathbb{R}_{+}^{p}, \quad \forall t \in \mathbb{R}_{+} .
$$

Define $z(t):=\mathrm{e}^{\gamma t} x(t)$ for $t \in \mathbb{R}_{+}$. A routine calculation using (1.1) shows that $z$ is a solution of

$$
\dot{z}(t)-(A+\gamma I) z(t) \in B \mathrm{e}^{\gamma t} F\left(\mathrm{e}^{-\gamma t} C z(t)\right), \quad z(0)=x^{0}, \quad t \in \mathbb{R}_{+} .
$$

Multiplying both sides of (2.28) by $-v^{T} C(A+\gamma I)^{-1}$ and invoking (2.27), we obtain the estimate

$$
\theta_{\gamma} v^{T} C z(t) \leq-v^{T} C(A+\gamma I)^{-1} z(t) \leq-v^{T} C(A+\gamma I)^{-1} x^{0} \quad \forall t \in \mathbb{R}_{+},
$$

for some $\theta_{\gamma}>0$, so that there exists $L>0$ such that

$$
\|C z(t)\| \leq L\left\|x^{0}\right\| \quad \forall t \in \mathbb{R}_{+} .
$$

Applying Lemma 2.1 to (2.28) with $D=\{0\}$ and $G(t, C x(t))=F(C x(t))$ shows that

$$
z(t)-\mathrm{e}^{(A+\gamma I) t} x^{0} \in \int_{0}^{t} \mathrm{e}^{(A+\gamma I)(t-\tau)} B \mathrm{e}^{\gamma \tau} F\left(\mathrm{e}^{-\gamma \tau} C z(\tau)\right) d \tau \quad \forall t \in \mathbb{R}_{+} .
$$

Invoking the boundedness of $C z$ and (A3) we see that

$$
\begin{aligned}
\|z(t)\| & \leq\left\|\mathrm{e}^{(A+\gamma I) t} x^{0}\right\|+\int_{0}^{t}\|\| \mathrm{e}^{(A+\gamma I)(t-\tau)} B \mathrm{e}^{\gamma \tau} F\left(\mathrm{e}^{-\gamma \tau} C z(\tau)\right) \| d \tau \\
& \leq\left(\left\|\mathrm{e}^{(A+\gamma I) t}\right\|+L R \int_{0}^{t}\left\|\mathrm{e}^{(A+\gamma I)(t-\tau)} B\right\| d \tau\right)\left\|x^{0}\right\| \quad \forall t \in \mathbb{R}_{+} .
\end{aligned}
$$

Since $\alpha(A+\gamma I)<0$ and $x^{0} \in \mathbb{R}_{+}^{n}$ was arbitrary, we conclude that there exists $\Gamma>0$ such that

$$
\|z(t)\| \leq \Gamma\left\|x^{0}\right\| \quad \forall t \in \mathbb{R}_{+}, \forall x^{0} \in \mathbb{R}_{+}^{n}
$$

and so

$$
\|x(t)\| \leq \Gamma \mathrm{e}^{-\gamma t}\left\|x^{0}\right\| \quad \forall t \in \mathbb{R}_{+}, \forall x^{0} \in \mathbb{R}_{+}^{n},
$$

as required.

In certain cases, the weighted one-norm estimate (2.9) itself implies that $F$ must satisfy (A3), which we formulate as the next lemma.

Lemma 2.7 Assume that $A, B, C$ and $F$ satisfy (A1) and (A2), and let $\mathbf{G}(s)=C(s I-$ $A)^{-1} B$. If (2.9), and at least one of the two conditions 
(a) $m=p$ and $\mathbf{G}(0)$ is irreducible;

(b) $B, C \neq 0, B$ has no zero columns and there exists $\Delta \in \mathbb{R}_{+}^{m \times p}$ such that $A+B \Delta C$ is irreducible;

hold, then $F$ satisfies (A3).

Proof Assume that (a) holds. By the Perron-Frobenius theorem, irreducibility of $\mathbf{G}(0)$ implies that there exists a strictly positive $v \in \mathbb{R}_{+}^{m}$ and $r>0$ such that

$$
v^{T} \mathbf{G}(0)=r v^{T} \quad \text { and so } \quad|\mathbf{G}(0) z|_{v}=r|z|_{v} \quad \forall z \in \mathbb{R}_{+}^{m}
$$

The above equality, combined with norm equivalence of all norms on $\mathbb{R}^{m}$, implies that there exists $\theta_{1}, \theta_{2}>0$ such that

$$
\theta_{1}\|z\| \leq\|\mathbf{G}(0) z\| \leq \theta_{2}|\mathbf{G}(0) z|_{v} \quad \forall z \in \mathbb{R}_{+}^{m}
$$

Thus, by (2.9) and (2.29)

$$
\theta_{1}\|w\| \leq\|\mathbf{G}(0) w\| \leq \theta_{2}|\mathbf{G}(0) w|_{v} \leq \theta_{2}|y|_{v} \leq c \theta_{2}\|y\| \quad \forall w \in F(y), \quad \forall y \in \mathbb{R}_{+}^{p},
$$

for some $c>0$, which demonstrates that (A3) holds with $R:=c \theta_{2} / \theta_{1}$.

Now assume that (b) holds. Suppose that $z \in \mathbb{R}_{+}^{m}$ is such that $\mathbf{G}(0) z=0$. Since $\alpha(A)<0$ we have that

$$
0=\mathbf{G}(0) z=-C A^{-1} B z=\int_{0}^{\infty} C \mathrm{e}^{(A+\delta I) t} \mathrm{e}^{-\delta t} B z d t \geq 0
$$

where $\delta>0$ is such that $0 \leq A+\delta I$ (such a $\delta$ exists as $A$ is Metzler). As the integrand is continuous and nonnegative, (2.30) implies that

$$
0=C \mathrm{e}^{(A+\delta I) t} B z=\sum_{k=0}^{\infty} \frac{t^{k}}{k !} C(A+\delta I)^{k} B z \quad \forall t \geq 0,
$$

whence,

$$
C(A+\delta I)^{k} B z=0 \quad \forall k \in \mathbb{N}_{0},
$$

and, therefore,

$$
C(A+\delta I+B \Delta C)^{k} B z=0 \quad \forall k \in \mathbb{N}_{0} .
$$

Let $c_{i}^{T} \neq 0$ and $b_{j}$ denote the $i$-th row and $j$-th column of $C$ and $B$, respectively, where we have used that $C \neq 0$. For each $r \in \underline{m}$, we see from (2.31) that

$$
c_{i}^{T}(A+\delta I+B \Delta C)^{k} b_{r} z_{r}=0 \quad \forall k \in \mathbb{N}_{0}
$$


As $b_{r} \neq 0$ for every $r \in \underline{m}$ and $A+\delta I+B \Delta C$ is irreducible, it follows from (2.32), by appropriate choices of $k \in \underline{n}$, that $z_{r}=0$. Therefore, we deduce that $z=0$ as $r \in \underline{m}$ was arbitrary and thus $\mathbf{G}(0)$ has no zero columns. Defining

$$
\beta=\min _{j \in \underline{m}}\left(\sum_{i=1}^{p}[\mathbf{G}(0)]_{i j}\right)>0,
$$

we obtain, for $z \in \mathbb{R}_{+}^{m}$,

$$
\|\mathbf{G}(0) z\|_{1}=\sum_{i=1}^{p}(\mathbf{G}(0) z)_{i}=\sum_{j=1}^{m}\left(\sum_{i=1}^{p}[\mathbf{G}(0)]_{i j}\right) z_{j} \geq \beta \sum_{j=1}^{m} z_{j}=\beta\|z\|_{1} .
$$

We deduce that (2.29) holds, for some $\theta_{1}, \theta_{2}>0$ which, when combined with (2.9) and using arguments identical to those used in the first part of the proof, establishes the claim.

The condition (2.10) is an intermediate between (2.9) and (2.11) which, as the next result shows, simplifies if more regularity assumptions are made on $F$.

Corollary 2.8 Assume that (A1)-(A3) hold and that $F$ is upper semi-continuous with closed values. If there exists a strictly positive $v \in \mathbb{R}_{+}^{p}$ such that

$$
|\mathbf{G}(0) w|_{v}<|y|_{v} \quad \forall w \in F(y) \quad \forall y \in \mathbb{R}_{+}^{p} \backslash\{0\},
$$

then, for all $x^{0} \in \mathbb{R}_{+}^{n}$, every global solution $x$ of (1.1) satisfies $x(t) \rightarrow 0$ as $t \rightarrow \infty$.

Proof We note that it is sufficient to show that (2.22) holds, because in this case the proof of the corollary may be completed by arguments identical to those used in the proof of statement (ii) of Theorem 2.5. To see that (2.22) holds, let $y_{k} \in \mathcal{M}$ and $w_{k} \in F\left(y_{k}\right)$ be such that

$$
v^{T} y_{k}-v^{T} \mathbf{G}(0) w_{k} \rightarrow \inf \left\{v^{T} \xi-v^{T} \mathbf{G}(0) w: w \in F(\xi), \xi \in \mathcal{M}\right\} \quad \text { as } k \rightarrow \infty,
$$

where $\mathcal{M}$ is given by (2.21). Since $y_{k} \in \mathcal{M}$ and $\mathcal{M}$ is compact, $\left(y_{k}\right)_{k \in \mathbb{N}}$ has a convergent subsequence, not relabelled, with limit $y_{*} \in \mathcal{M}$, hence $y_{*} \neq 0$. The upper semi-continuity of $F$ means that we may choose another subsequence of $\left(y_{k}\right)_{k \in \mathbb{N}}$, again not relabelled, such that

$$
w_{k} \in F\left(y_{k}\right) \subseteq F\left(y_{*}\right)+B(0,1 / k)
$$

where $B(x, r) \subseteq \mathbb{R}^{m}$ denotes the open ball centred at $x$ with radius $r>0$. Assumption (A3) implies that $F\left(y_{*}\right)$ is bounded and so, by $(2.34),\left(w_{k}\right)_{k \in \mathbb{N}}$ is bounded, and hence has a convergent subsequence, not relabelled, with limit $w_{*}$. Necessarily, from (2.34) we see that $w_{*} \in \overline{F\left(y_{*}\right)}=F\left(y_{*}\right)$, as $F$ is assumed to be closed valued. We conclude that 


$$
\begin{aligned}
\inf \left\{v^{T} \xi-v^{T} \mathbf{G}(0) w: w \in F(\xi), \xi \in \mathcal{M}\right\} & =\lim _{k \rightarrow \infty}\left[v^{T} y_{k}-v^{T} \mathbf{G}(0) w_{k}\right] \\
& =v^{T} y_{*}-v^{T} \mathbf{G}(0) w_{*}>0,
\end{aligned}
$$

by $(2.33)$ as $y_{*} \neq 0$, showing that (2.22) holds.

Although formulated for autonomous problems, Theorem 2.5 readily extends to the non-autonomous differential inclusion (2.5) (still with $D=\{0\}$ ), provided the conditions in Theorem 2.5 hold uniformly in time. We formulate these claims in the next corollary.

Corollary 2.9 Given the Lur'e inclusion (2.5) with $D=\{0\}$, assume that $A, B, C$ and G satisfy (A1)-(A3). Define

$$
F: \mathbb{R}_{+}^{p} \rightarrow P_{0}\left(\mathbb{R}_{+}^{m}\right), \quad F(y):=\bigcup_{t \geq 0} G(t, y) .
$$

If F given by (2.35) satisfies the conditions (2.9)-(2.11) in Theorem 2.5, then the conclusions of Theorem 2.5 hold for every global solution of (2.5) with $D=\{0\}$.

\subsection{Forced systems}

We next consider the system of forced Lur'e differential inclusions (1.3), where $D$ : $\mathbb{R}_{+} \rightarrow P_{0}\left(\mathbb{R}_{+}^{n}\right)$. We say that $D: \mathbb{R}_{+} \rightarrow P_{0}\left(\mathbb{R}_{+}^{n}\right)$ is (essentially) locally bounded or (essentially) bounded if

$$
\underset{\tau \in[0, t]}{\operatorname{ess} \sup }\|\mid D(\tau)\| \|<\quad \forall t \in \mathbb{R}_{+} \quad \text { or } \underset{\tau \in[0, \infty)}{\operatorname{ess} \sup }\||D(\tau) \||<\infty,
$$

respectively.

With regards to the existence of solutions of (1.3), we refer to, for example [26, Chap. 3, Sections 5-6] or [67, Theorems 1.1, 1.3, pp. 30-31]. The following result is based on [26, Theorem 5.2].

Proposition 2.10 Given the forced Lur'e inclusion (1.3), assume that:

(i) (A1)-(A3) hold;

(ii) $F$ has closed, convex values, $F(t, \cdot)$ is upper semi-continuous and $F(\cdot, x)$ is measurable;

(iii) D is measurable, locally bounded with closed, convex values.

Then, for every $x^{0} \in \mathbb{R}_{+}^{n}$, (1.3) has a global solution, and every solution may be extended to a global solution. Further, every solution $x$ satisfies $x(t) \in \mathbb{R}_{+}^{n}$ for all $t$ where $x(t)$ is defined.

Proof Existence of global solutions follows from an application of [26, Theorem 5.2] and [26, Corollary 5.2, p. 58] ensures that solutions may be extended to global solutions. Lemma 2.2 ensures that every solution is nonnegative. 
Assumption (A3) implies that $F(0)=\{0\}$ and hence $x=0, D=\{0\}$ is a solution of (1.3) with $x^{0}=0$, which we shall hereafter refer to as the zero equilibrium pair. We proceed to state and prove the main result of the present section, namely that the assumptions made in statement (iii) of Theorem 2.5 are sufficient for the zero equilibrium pair of (1.3) to be so-called exponentially ISS. The proof is similar to that of statement (iii) of Theorem 2.5, and uses a so-called exponential weighting argument, see [36].

As will become apparent in the proof of Corollary 2.12, it is convenient in the next result to impose no assumption on sign of the disturbance term $D$, but instead assume that the state $x$ remains nonnegative.

Theorem 2.11 Given the forced Lur'e inclusion (1.3), assume that (A1)-(A3) hold. If there exist a strictly positive $v \in \mathbb{R}_{+}^{p}$ and $\rho \in(0,1)$ such that $(2.11)$ holds, then there exists $\Gamma, \gamma>0$ such that, for all $x^{0} \in \mathbb{R}_{+}^{n}$, all locally bounded $D: \mathbb{R}_{+} \rightarrow P_{0}\left(\mathbb{R}^{n}\right)$ and every global nonnegative solution $x$ of (1.3),

$$
\|x(t)\| \leq \Gamma\left(\mathrm{e}^{-\gamma t}\left\|x^{0}\right\|+\underset{\tau \in[0, t]}{\operatorname{ess} \sup }\|D(\tau)\|\right) \quad \forall t \in \mathbb{R}_{+} .
$$

If $D$ is nonnegative-valued, that is $D(t) \subseteq \mathbb{R}_{+}^{n}$ for almost all $t \geq 0$, then the hypotheses of Theorem 2.11 ensure that (2.36) holds for every global solution $x$ of (1.3). The inequality (2.36) is the definition of exponential ISS of the zero equilibrium pair (in the current set-valued setting).

Proof of Theorem 2.11 Fix $x^{0} \in \mathbb{R}_{+}^{n}$ and $D: \mathbb{R}_{+} \rightarrow P_{0}\left(\mathbb{R}^{n}\right)$ as above and let $x$ be a global, nonnegative solution of (1.3). Let $\varepsilon, \gamma>0$ be such that $\rho+\varepsilon<1$ and $\alpha(A+\gamma I)<0$. Defining $z(t):=\mathrm{e}^{\gamma t} x(t)$ for $t \geq 0$, which is also nonnegative, an elementary calculation using (1.3) shows that $z$ is a solution of

$$
\dot{z}(t)-(A+\gamma I) z(t) \in B \mathrm{e}^{\gamma t} F\left(\mathrm{e}^{-\gamma t} C z(t)\right)+\mathrm{e}^{\gamma t} D(t), \quad z(0)=x^{0}, \quad t \in \mathbb{R}_{+} .
$$

Multiplying both sides of the above by $v^{T} C(-\gamma I-A)^{-1}$ yields that, for almost all $t \in \mathbb{R}_{+}$

$$
v^{T} C(-\gamma I-A)^{-1} \dot{z}(t)+v^{T} C z(t) \in v^{T} \mathbf{G}(-\gamma) \mathrm{e}^{\gamma t} F\left(\mathrm{e}^{-\gamma t} C z(t)\right)+H(t)
$$

where

$$
H(t):=v^{T} C(-\gamma I-A)^{-1} \mathrm{e}^{\gamma t} D(t) \quad \forall t \in \mathbb{R}_{+} .
$$

Using arguments similar to those involved in the derivation of (2.26), we see that for sufficiently small $\gamma>0$

$$
v^{T} \mathbf{G}(-\gamma) \mathrm{e}^{\gamma t} F\left(\mathrm{e}^{-\gamma t} C z(t)\right) \subseteq\left[0,(\rho+\varepsilon) v^{T} C z(t)\right] \quad \forall t \in \mathbb{R}_{+},
$$

whence, for almost all $t \in \mathbb{R}_{+}$

$$
v^{T} \mathbf{G}(-\gamma) \mathrm{e}^{\gamma t} F\left(\mathrm{e}^{-\gamma t} C z(t)\right)+H(t) \subseteq\left[0,(\rho+\varepsilon) v^{T} C z(t)\right]+H(t)
$$


Setting $\delta=1-(\rho+\varepsilon) \in(0,1)$ and

$$
\left.\begin{array}{l}
E(t):=\left[-v^{T} C z(t),-\delta v^{T} C z(t)\right] \\
a(t):=-\delta v^{T} \int_{0}^{t} C z(\tau) d \tau \\
\xi(t):=\kappa \underset{\tau \in[0, t]}{\operatorname{ess} \sup }\|D(\tau)\| \int_{0}^{t} \mathrm{e}^{\gamma \tau} d \tau
\end{array}\right\} \forall t \in \mathbb{R}_{+},
$$

where $\kappa:=\left\|v^{T} C(\gamma I+A)^{-1}\right\|>0$, it follows from (2.38)-(2.40) that

$$
v^{T} C(-\gamma I-A)^{-1} \dot{z}(\tau) \in E(\tau)+H(\tau) \text { for almost all } \tau \in \mathbb{R}_{+},
$$

so that

$$
v^{T} C(-\gamma I-A)^{-1} z(t)-v^{T} C(-\gamma I-A)^{-1} x^{0} \in \int_{0}^{t}(E(\tau)+H(\tau)) d \tau \quad \forall t \in \mathbb{R}_{+} .
$$

Choose $\sigma \in(0, \delta)$, and note that by definition of $a$ and monotonicity of the integral

$$
\sigma \underset{\sim}{v}\|C z\|_{L^{1}(0, t)}+a(t) \leq \sigma \int_{0}^{t} v^{T} C z(\tau) d \tau+a(t) \leq 0 \quad \forall t \in \mathbb{R}_{+} .
$$

Be definition of $a$ and $\xi$, it follows from (2.42) that

$$
\begin{aligned}
v^{T} C(-\gamma I-A)^{-1} z(t)+\sigma \underset{\sim}{v}\|C z\|_{L^{1}(0, t)} \leq & v^{T} C(-\gamma I-A)^{-1} x^{0}+\sigma \underset{\sim}{v}\|C z\|_{L^{1}(0, t)} \\
& +a(t)+\xi(t) \\
\leq & v^{T} C(-\gamma I-A)^{-1} x^{0}+\xi(t) \quad \forall t \in \mathbb{R}_{+},
\end{aligned}
$$

by (2.43). Now, as $0 \leq v^{T} C(-\gamma I-A)^{-1} z(t)$, appealing to (2.41) yields that

$$
\|C z\|_{L^{1}(0, t)} \leq K_{1}\left\|x_{0}\right\|+K_{2} \mathrm{e}^{\gamma t} \sup _{\tau \in[0, t]}\|D(\tau)\| \quad \forall t \in \mathbb{R}_{+},
$$

for some positive constants $K_{1}$ and $K_{2}$, which are independent of $t, D$ and $x^{0}$.

An application of Lemma 2.1 to (2.37) implies that

$$
z(t)-\mathrm{e}^{(A+\gamma I) t} x^{0} \in \int_{0}^{t} \mathrm{e}^{(A+\gamma I)(t-\tau)}\left[B \mathrm{e}^{\gamma \tau} F\left(\mathrm{e}^{-\gamma \tau} C z(\tau)\right)+\mathrm{e}^{\gamma \tau} D(\tau)\right] d \tau \quad \forall t \in \mathbb{R}_{+} .
$$

Invoking (A3) and $\alpha(A+\gamma I)<0$, we estimate $\|z(t)\|$ using the above inclusion as follows 


$$
\begin{aligned}
\|z(t)\| & \leq K_{3}\left\|x^{0}\right\|+K_{4}\|C z\|_{L^{1}(0, t)}+K_{5} \mathrm{e}^{\gamma t} \sup _{\tau \in[0, t]}\|D(\tau)\| \\
& \leq\left(K_{3}+K_{4} K_{1}\right)\left\|x^{0}\right\|+\left(K_{4} K_{2}+K_{5}\right) \mathrm{e}^{\gamma t} \underset{\tau \in[0, t]}{\operatorname{ess} \sup }\|D(\tau)\| \| \quad \forall t \in \mathbb{R}_{+},
\end{aligned}
$$

by (2.44), for some $K_{3}, K_{4}, K_{5}>0$ which are independent of $t, D$ and $x^{0}$. Thus,

$$
\|x(t)\| \leq \Gamma\left(\mathrm{e}^{-\gamma t}\left\|x^{0}\right\|+\underset{\tau \in[0, t]}{\operatorname{ess} \sup }\|D(\tau)\|\right) \quad \forall t \in \mathbb{R}_{+}
$$

where $\Gamma:=\max \left\{K_{3}+K_{4} K_{1}, K_{4} K_{2}+K_{5}\right\}$, completing the proof.

The next corollary is a so-called ISS with bias result (see $[36,48]$ ) which states that if the condition (2.11) fails on a bounded set, then solutions of (1.3) (which include those of (1.1)) still admit uniform estimates of the form (2.36), but with an additional positive constant term. We note that ISS with bias is closely related to the concept of input-to-state practical stability (ISpS), see [68,69]. Although ISpS applies to more general nonlinear forced control systems, a difference is that it does not typically specify the form of the additional constant, denoted $\beta$ in the bounds below.

Corollary 2.12 Given the forced differential Lur'e inclusion (1.3), assume that (A1)(A3) hold. If there exist a strictly positive $v \in \mathbb{R}_{+}^{p}, \rho \in(0,1)$ and $\Theta \geq 0$ such that

$$
|\mathbf{G}(0) w|_{v} \leq \rho|y|_{v} \quad \forall w \in F(y) \quad \forall y \in \mathbb{R}_{+}^{p},\|y\| \geq \Theta,
$$

then there exist $\Gamma, \gamma>0$ such that, for all $x^{0} \in \mathbb{R}_{+}^{n}$ and all locally bounded $D$ : $\mathbb{R}_{+} \rightarrow P_{0}\left(\mathbb{R}_{+}^{n}\right)$, every global solution $x$ of (1.3) satisfies

$$
\|x(t)\| \leq \Gamma\left(\mathrm{e}^{-\gamma t}\left\|x^{0}\right\|+\underset{\tau \in[0, t]}{\operatorname{ess} \sup }\|D(\tau)\|+\beta\right) \quad \forall t \in \mathbb{R}_{+} .
$$

Here

$$
\beta:=\|B\| \sup _{\|y\| \leq \theta}\left(\sup _{w \in F(y)}(\operatorname{dist}(w, S(y)))\right),
$$

and

$$
S(y):=\left\{w \in F(y):|\mathbf{G}(0) w|_{v} \leq \rho|y|_{v}\right\} \subseteq F(y) .
$$

The number $\beta$ in (2.47) seeks to capture the extent to which the inequality in (2.45) is violated on the set $\left\{y \in \mathbb{R}_{+}^{p}:\|y\|<\Theta\right\}$. Observe that if $\Theta=0$, then $\beta=0$ and the conclusions of Theorem 2.11 and Corollary 2.12 coincide.

Proof of Corollary 2.12 Define $H: \mathbb{R}_{+}^{p} \rightarrow P_{0}\left(\mathbb{R}_{+}^{m}\right)$ by

$$
H(y)= \begin{cases}F(y) & \|y\| \geq \Theta \\ S(y) & \|y\|<\Theta\end{cases}
$$


where $S(y)$ is given by (2.48), so that $H$ satisfies (A3). For given $x^{0} \in \mathbb{R}_{+}^{n}$ and $D: \mathbb{R}_{+} \rightarrow P_{0}\left(\mathbb{R}_{+}^{n}\right)$, let $x$ denote a global solution of (1.3). As $D(t) \subseteq \mathbb{R}_{+}^{n}$ for almost all $t \geq 0$, we have that $x \geq 0$ and thus $x$ is also a global nonnegative solution of

$$
\dot{x}-A x \in B H(C x)+B[F(C x)-H(C x)]+D=B H(C x)+E, \quad x(0)=x^{0},
$$

where $E:=B[F(C x)-H(C x)]+D$. We seek to apply Theorem 2.11 to (2.50), with $F$ and $D$ in (1.3) replaced by $H$ and $E$, respectively. Although it is possible that $E(t) \nsubseteq \mathbb{R}_{+}^{n}$ for some $t \geq 0$, since $x$ is nonnegative it suffices to verify that $E$ is locally bounded. We consider two exhaustive cases: if $\|C x(t)\| \geq \Theta$, then

$$
\underset{\tau \in[0, t]}{\operatorname{ess} \sup }\|E(t)\|=\underset{\tau \in[0, t]}{\operatorname{ess} \sup }\|D(\tau)\|<\infty,
$$

by construction. Alternatively, if $\|C x(t)\|<\Theta$

$$
\begin{aligned}
\underset{\tau \in[0, t]}{\operatorname{ess} \sup }\|E(t)\| & \leq \sup _{t \in[0, \tau]}\|B[F(C x(t))-H(C x(t))]\|\|+\underset{\tau \in[0, t]}{\operatorname{ess} \sup }\| D(\tau) \| \\
& \leq \beta+\underset{\tau \in[0, t]}{\operatorname{ess} \sup }\|D(\tau)\|<\infty
\end{aligned}
$$

Therefore, the hypotheses of Theorem 2.11 hold which, when combined with (2.51), yield the existence of $\Gamma, \gamma>0$ such that $x$ satisfies the estimate (2.46).

\subsection{Positive Lur'e differential equations and inequalities}

As indicated in the Introduction, the stability (or otherwise) of equilibria of Lur'e differential equations, or simply Lur'e systems, has been the focus of much attention in the control theory literature. The forced positive Lur'e system

$$
\dot{x}(t)=A x(t)+B g(t, C x(t))+d(t), \quad x(0)=x^{0}, \quad t \in \mathbb{R}_{+},
$$

where $g: \mathbb{R}_{+} \times \mathbb{R}_{+}^{p} \rightarrow \mathbb{R}_{+}^{m}$ and $d: \mathbb{R}_{+} \rightarrow \mathbb{R}_{+}^{n}$, is a special case of the forced positive Lur'e inclusion (1.3) with

$$
F(y):=\bigcup_{t \in \mathbb{R}_{+}}\{g(t, y)\} \quad \forall y \in \mathbb{R}_{+}^{p} \text { and } D(t):=\{d(t)\} \quad \forall t \in \mathbb{R}_{+} .
$$

In certain applications, the so-called forced positive Lur'e inequality

$$
0 \leq \dot{x}(t)-A x(t) \leq B g(t, C x(t))+d(t), \quad x(0)=x^{0}, \quad t \in \mathbb{R}_{+},
$$

is also of interest. Note that an absolutely continuous function $x: \mathbb{R}_{+} \rightarrow \mathbb{R}^{n}$ is a solution of (2.53) if, and only if, $x$ is a solution of (1.3) with

$$
F(y):=\bigcup_{t \in \mathbb{R}_{+}}[0, g(t, y)] \quad \forall y \in \mathbb{R}_{+}^{p} \text { and } D(t):=[0, d(t)] \forall t \in \mathbb{R}_{+} .
$$


In both of the special cases above, the set-valued map $F$ satisfies assumption (A3) if, and only if, there exists $R>0$ such that

$$
\|g(t, y)\| \leq R\|y\| \quad \forall t \in \mathbb{R}_{+}, \quad \forall y \in \mathbb{R}_{+}^{p} .
$$

We note that (2.52) is a particular case of (2.53), and so focus attention on (2.53). The following corollary is an immediate consequence of Theorems 2.5, 2.11 and Corollary 2.12 .

Corollary 2.13 Given the forced positive Lur'e inequality (2.53), assume that (A1) and (A2) hold and that $g$ satisfies (2.54).

(i) If $d=0$ and there exists a strictly positive $v \in \mathbb{R}_{+}^{p}$ such that

$$
|\mathbf{G}(0) g(t, y)|_{v} \leq|y|_{v} \quad \forall t \in \mathbb{R}_{+}, \quad \forall y \in \mathbb{R}_{+}^{p},
$$

then there exists $\Gamma>0$ such that, for all $x^{0} \in \mathbb{R}_{+}^{n}$, every global solution $x$ of (2.53) satisfies

$$
\|x(t)\| \leq \Gamma\left\|x^{0}\right\| \quad \forall t \in \mathbb{R}_{+} .
$$

(ii) If $d=0$ and there exist a strictly positive $v \in \mathbb{R}_{+}^{p}$ and a lower semi-continuous function $e: \mathbb{R}_{+}^{p} \rightarrow \mathbb{R}_{+}$such that

$$
e(y)>0 \text { and }|\mathbf{G}(0) g(t, y)|_{v}+e(y) \leq|y|_{v} \quad \forall t \in \mathbb{R}_{+}, \quad \forall y \in \mathbb{R}_{+}^{p} \backslash\{0\}
$$

then, for all $x^{0} \in \mathbb{R}_{+}^{n}$, every global solution $x$ of (2.53) satisfies $x(t) \rightarrow 0$ as $t \rightarrow \infty$.

(iii) If there exist a strictly positive $v \in \mathbb{R}_{+}^{p}, \rho \in(0,1)$ and $\Theta \geq 0$ such that

$$
|\mathbf{G}(0) g(t, y)|_{v} \leq \rho|y|_{v} \quad \forall t \in \mathbb{R}_{+}, \quad \forall y \in \mathbb{R}_{+}^{p}\|y\| \geq \Theta,
$$

then there exist $\Gamma, \gamma>0$ such that, for all $x^{0} \in \mathbb{R}_{+}^{n}$ and all $d \in L_{\mathrm{loc}}^{\infty}\left(\mathbb{R}_{+} ; \mathbb{R}_{+}^{n}\right)$, every global solution $x$ of (2.53) satisfies

$$
\|x(t)\| \leq \Gamma\left(\mathrm{e}^{-\gamma t}\left\|x^{0}\right\|+\|d(\tau)\|_{L^{\infty}(0, t)}+\beta\right) \quad \forall t \in \mathbb{R}_{+} .
$$

Here

$$
\beta=\beta(\Theta)=\|B\| \sup _{\|y\| \leq \Theta}\left(\sup _{t \geq 0}(\operatorname{dist}(g(t, y), T(t, y)))\right),
$$

and

$$
T(t, y):=\left\{w \in[0, g(t, y)] \subseteq \mathbb{R}_{+}^{m}:|\mathbf{G}(0) w|_{v} \leq \rho|y|_{v}\right\}
$$


The following remark provides some commentary on the above corollary.

Remark 2.14 As the conditions (2.55)-(2.57) are assumed to hold uniformly in the first variable, for ease of presentation the following comments suppress this variable.

(a) In the situation where $m=p=1$ and $g$ is continuous, the conditions (2.55), (2.56) and (2.57) (the latter with $\Theta=0$ ) simplify to:

$\mathbf{G}(0) g(y) \leq y, \quad \mathbf{G}(0) g(y)<y$ (for $y>0$ ) and $\mathbf{G}(0) g(y) \leq \rho y \quad \forall y \in \mathbb{R}_{+}$, respectively.

(b) When $m=p$, a sufficient condition for (2.55) or (2.57) are the inequalities

$$
\|\mathbf{G}(0)\|_{v}\|g\|_{v} \leq 1 \text { or }\|\mathbf{G}(0)\|_{v}\|g\|_{v}<1
$$

respectively, where

$$
\|\mathbf{G}(0)\|_{v}:=\sup _{\substack{\xi \in \mathbb{R}_{+}^{m} \\ \xi \neq 0}} \frac{|\mathbf{G}(0) \xi|_{v}}{|\xi|_{v}} \text { and }\|g\|_{v}:=\sup _{\substack{\xi \in \mathbb{R}_{+}^{m} \\ \xi \neq 0}} \frac{|g(\xi)|_{v}}{|\xi|_{v}}
$$

are the induced $v$-norms. The inequalities in (2.58) are reminiscent of classical small-gain conditions, only here formed in the induced $v$-norm. We note that in general, $\|\mathbf{G}(0)\|_{v} \neq\|\mathbf{G}(0)\|_{2}=\|\mathbf{G}\|_{H^{\infty}}$, where the final equality is a property enjoyed by linear positive systems; see, for example [24, Theorem 5].

(c) The conclusions of statements (i) and (ii) of Corollary 2.13 are similar to those in [58, Theorem 7.2] or [1, Theorem 5.6, p. 156], where a linear dissipativity theory approach to the absolute stability of positive Lur'e systems is taken. In [58, Theorem 7.2] the authors assume that the pair $(C, A)$ is observable, and that the nonlinearity $g$ satisfies $0 \leq g(y) \leq M y$ for all $y \in \mathbb{R}_{+}^{p}$, for some nonnegative matrix $M \in \mathbb{R}_{+}^{m \times p}$. Further, it is assumed that the triple $(A, B, C)$ is exponentially linearly dissipative with respect to the supply rate $s(u, y)=\mathbb{1}^{T} u-\mathbb{1}^{T} M y$, which is equivalent to the inequality

$$
\mathbb{1}^{T} M \mathbf{G}(0) \ll \mathbb{1}^{T}
$$

The inequality (2.59) is itself equivalent to $|M \mathbf{G}(0)|_{\mathbb{1}}=\|M \mathbf{G}(0)\|_{1}<1$ and may be interpreted as a small-gain condition in the induced the one-norm. Although not directly comparable, our norm conditions in (i)-(iii) are more general as they allow a small-gain condition in a weighted one-norm induced by any strictly positive vector, not just the usual one-norm, see Example 4.3. Finally, we remark that [58] focusses on unforced systems only.

\section{Discrete-time systems}

In this section we turn attention to the systems of forced Lur'e difference inclusions (1.4) with properties: 
(B1) $(A, B, C) \in \mathbb{R}_{+}^{n \times n} \times \mathbb{R}_{+}^{n \times m} \times \mathbb{R}_{+}^{p \times n}, D: \mathbb{N}_{0} \rightarrow P_{0}\left(\mathbb{R}_{+}^{n}\right)$ and $F: \mathbb{R}_{+}^{p} \rightarrow$ $P_{0}\left(\mathbb{R}_{+}^{m}\right)$

(B2) $r(A)<1$;

where $F$ is also assumed to satisfy (A3). Given $x^{0} \in \mathbb{R}_{+}^{n}$ and $D: \mathbb{N}_{0} \rightarrow P_{0}\left(\mathbb{R}_{+}^{n}\right)$, a function $x: \mathbb{N}_{0} \rightarrow \mathbb{R}_{+}^{n}$ satisfying (1.4) is called a solution of (1.4), the guaranteed existence of which is not a concern in discrete-time. As with the continuous-time setting, assumption (A3) implies that $x=0, D=\{0\}$ is a solution of (1.4) with $x^{0}=0$ which we refer to as the zero equilibrium pair. We comment that "loopshifting", see (2.7), is also possible in discrete-time, particularly if (B2) fails, by replacing $A$ and $F$ by $A+B K C$ and $y \mapsto F(y)-K y$, respectively, for $K \in \mathbb{R}^{m \times p}$.

Our main result of this section contains a series of global stability (when $D=$ $\{0\})$ and ISS results for (1.4), formulated in terms of induced weighted one-norm constraints. As before, let $\mathbf{G}$ denote the transfer function of the triple $(A, B, C)$ with $\mathbf{G}(1)=C(I-A)^{-1} B$. Assumption $(\mathbf{B} 2)$ implies that $\mathbf{G}(1)$ is well-defined and together with (B1) implies that $\mathbf{G}(1) \in \mathbb{R}_{+}^{p \times m}$.

Theorem 3.1 Given the Lur'e difference inclusion (1.4), assume that (B1), (B2) and (A3) hold.

(i) If $D=\{0\}$ and there exists a strictly positive $v \in \mathbb{R}_{+}^{p}$ such that

$$
|\mathbf{G}(1) w|_{v} \leq|y|_{v} \quad \forall w \in F(y) \quad \forall y \in \mathbb{R}_{+}^{p},
$$

then there exists $\Gamma>0$ such that, for all $x^{0} \in \mathbb{R}_{+}^{n}$, every solution $x$ of (1.1) satisfies

$$
\|x(t)\| \leq \Gamma\left\|x^{0}\right\| \quad \forall t \in \mathbb{N}_{0} .
$$

(ii) If $D=\{0\}$ and there exist a strictly positive $v \in \mathbb{R}_{+}^{p}$ and a lower semi-continuous function $e: \mathbb{R}_{+}^{p} \rightarrow \mathbb{R}_{+}$such that

$$
e(y)>0 \text { and }|\mathbf{G}(1) w|_{v}+e(y)<|y|_{v} \forall w \in F(y) \quad \forall y \in \mathbb{R}_{+}^{p} \backslash\{0\},
$$

then, for all $x^{0} \in \mathbb{R}_{+}^{n}$, every solution $x$ of (1.1) satisfies $x(t) \rightarrow 0$ as $t \rightarrow \infty$.

(iii) If there exist a strictly positive $v \in \mathbb{R}_{+}^{p}$ and $\rho \in(0,1)$ such that

$$
|\mathbf{G}(1) w|_{v} \leq \rho|y|_{v} \quad \forall w \in F(y) \quad \forall y \in \mathbb{R}_{+}^{p},
$$

then there exist $\Gamma>0$ and $\gamma \in(0,1)$ such that, for all $x^{0} \in \mathbb{R}_{+}^{n}$ and all $D: \mathbb{N}_{0} \rightarrow P_{0}\left(\mathbb{R}_{+}^{n}\right)$, every solution $x$ of (1.1) satisfies

$$
\|x(t)\| \leq \Gamma\left(\gamma^{t}\left\|x^{0}\right\|+\max _{\tau \in \underline{t-1}}\|D(\tau)\|\right) \quad \forall t \in \mathbb{N} .
$$

Statements (i), (ii) and (iii) of Theorem 3.1 imply that, with $D=\{0\}$, the zero equilibrium of (1.4) is stable in the large, globally asymptotically stable and globally exponentially stable, respectively. Moreover, statement (iii) implies that the zero equilibrium pair of (1.4) is exponentially ISS. 
Proof of Theorem 3.1 Throughout the proof let $x$ denote a solution of (1.4) for given $x^{0} \in \mathbb{R}_{+}^{n}$.

(i): The inequality (3.1) implies that

$$
v^{T} \mathbf{G}(1) w \in\left[0, v^{T} y\right] \quad \forall w \in F(y) \quad \forall y \in \mathbb{R}_{+}^{p} .
$$

Multiplying both sides of (1.4) by $v^{T} C(I-A)^{-1}$, subtracting $v^{T} C(I-A)^{-1} x(t)$ and invoking (3.4) yields

$$
\begin{aligned}
v^{T} C(I-A)^{-1} x(t+1)-v^{T} C(I-A)^{-1} x(t) & \in v^{T} C(I-A)^{-1}[A x(t)+B F(C x(t))] \\
& -v^{T} C(I-A)^{-1} x(t) \\
& \subseteq-v^{T} C x(t)+\left[0, v^{T} C x(t)\right]=\left[-v^{T} C x(t), 0\right],
\end{aligned}
$$

so that

$$
v^{T} C(I-A)^{-1} x(t+1)-v^{T} C(I-A)^{-1} x(t) \leq 0 \quad \forall t \in \mathbb{N}_{0} .
$$

Furthermore, as $A \geq 0$ and $r(A)<1$,

$$
C z \leq C\left(\sum_{k=0}^{\infty} A^{k}\right) z=C(I-A)^{-1} z \quad \forall z \in \mathbb{R}_{+}^{n},
$$

and hence, arguing inductively in (3.5), we see that

$$
v^{T} C x(t) \leq v^{T} C(I-A)^{-1} x(t) \leq v^{T} C(I-A)^{-1} x^{0} \quad \forall t \in \mathbb{N}_{0} .
$$

Consequently, there exists $P>0$ such that

$$
\|C x(t)\| \leq P\left\|x^{0}\right\| \quad \forall t \in \mathbb{N}_{0} .
$$

As $r(A)<1$, statement (i) now follows from the variation of parameters inclusion

$$
x(t)-A^{t} x^{0} \in \sum_{j=0}^{t-1} A^{t-1-j} B F(C x(j)) \quad t \in \mathbb{N},
$$

(which is easily established from (1.4) via induction on $t$ ), the estimate (3.6) and (A3). (ii): Our hypotheses ensure that statement (i) holds, in particular meaning that every global solution is bounded. It is sufficient to show that $C x(t) \rightarrow 0$ as $t \rightarrow \infty$, as then $x(t) \rightarrow 0$ as $t \rightarrow 0$ by (3.7), (B2) and (A3). To that end, fix $x^{0} \in \mathbb{R}_{+}^{n} \backslash\{0\}$ and, seeking a contradiction, suppose that $C x(t) \nrightarrow \rightarrow 0$ as $t \rightarrow \infty$, implying that there exists $\left(t_{k}\right)_{k \in \mathbb{N}_{0}} \subseteq \mathbb{N}$ with $t_{k} \nearrow \infty$ as $k \rightarrow \infty$ and $\varepsilon>0$ such that

$$
\varepsilon \leq\left\|C x\left(t_{k}\right)\right\| \quad \forall k \in \mathbb{N}_{0} .
$$


Invoking statement (i), we conclude further that

$$
\varepsilon \leq\left\|C x\left(t_{k}\right)\right\| \leq \Gamma\|C\|\left\|x^{0}\right\| \quad \forall k \in \mathbb{N}_{0},
$$

and so $C x\left(t_{k}\right) \in \mathcal{M}$ for all $k \in \mathbb{N}_{0}$, with $\mathcal{M}$ defined as in (2.21). We claim that there exists $\eta>0$ such that

$$
\inf _{\substack{\xi \in \mathcal{M} \\ w \in F(\xi)}}\left[v^{T} \xi-v^{T} \mathbf{G}(1) w\right] \geq \eta
$$

The proof of (3.8) is identical to that of (2.22), and hence is omitted.

Next note that, by (3.5), the nonnegative sequence $\left(v^{T} C(I-A)^{-1} x(t)\right)_{t \in \mathbb{N}_{0}}$ is non-increasing and thus convergent. We now estimate that

$$
\begin{aligned}
v^{T} C(I-A)^{-1} x\left(t_{k}\right)-v^{T} C(I-A)^{-1} x\left(t_{k}+1\right) & \in v^{T} C x\left(t_{k}\right)-v^{T} \mathbf{G}(1) F\left(C x\left(t_{k}\right)\right) \\
& \subseteq[\eta, \infty),
\end{aligned}
$$

where the final inclusion follows from (3.8). As a consequence,

$$
v^{T} C(I-A)^{-1} x\left(t_{k}\right)-v^{T} C(I-A)^{-1} x\left(t_{k}+1\right) \geq \eta \quad \forall k \in \mathbb{N}_{0},
$$

which contradicts the convergence of $\left(v^{T} C(I-A)^{-1} x(t)\right)_{t \in \mathbb{N}_{0}}$.

(iii): Fix $x^{0} \in \mathbb{R}_{+}^{n}$, locally bounded $D: \mathbb{N}_{0} \rightarrow P_{0}\left(\mathbb{R}_{+}^{n}\right)$ and let $\varepsilon>0$ be such that $\kappa:=\rho+\varepsilon<1$. Since $r(A)<1$ and $\mathbf{G}$ is continuous at 1 , there exists $\delta \in(0,1)$ such that

$$
\frac{1}{1+\delta}<\gamma<1 \Rightarrow r(A / \gamma)<1 \text { and }\|\mathbf{G}(\gamma)-\mathbf{G}(1)\|<\frac{\varepsilon c_{1}}{R c_{2}}
$$

where $c_{1}, c_{2}>0$ are the constants in (2.24). Thus, for fixed $\gamma \in(1 /(1+\delta), 1)$ we note that $\mathbf{G}(\gamma) \geq 0$ and, further, for $y \in \mathbb{R}_{+}^{p}$ and $w \in F(y)$

$$
\begin{aligned}
|\mathbf{G}(\gamma) w|_{v} & =v^{T} \mathbf{G}(\gamma) w=v^{T}[\mathbf{G}(\gamma)-\mathbf{G}(1)] w+|\mathbf{G}(1) w|_{v} \\
& \leq c_{2}\|\mathbf{G}(\gamma)-\mathbf{G}(1)\|\|w\|+\rho|y|_{v} \leq \frac{\varepsilon c_{1}}{R}\|w\|+\rho|y|_{v} \\
& \leq \kappa|y|_{v},
\end{aligned}
$$

where we have used (A3), (2.24), (3.2) and (3.9). Multiplying both sides of the above inequality by $\gamma^{-t}$, and taking $y=\gamma^{t} \xi$ for $\xi \in \mathbb{R}_{+}^{p}$, we see that

$$
\left|\gamma^{-t} \mathbf{G}(\gamma) w\right|_{v} \leq \gamma^{-t} \cdot \kappa\left|\gamma^{t} \xi\right|_{v}=\kappa|\xi|_{v} \quad \forall w \in F\left(\gamma^{t} \xi\right) \quad \forall \xi \in \mathbb{R}_{+}^{p} \quad \forall t \in \mathbb{N}_{0}
$$

or, equivalently,

$$
v^{T} \gamma^{-t} \mathbf{G}(\gamma) F\left(\gamma^{t} \xi\right) \subseteq\left[0, \kappa v^{T} \xi\right] \quad \forall \xi \in \mathbb{R}_{+}^{p} \quad \forall t \in \mathbb{N}_{0}
$$


Define $z(t):=\gamma^{-t} x(t)$ and $H(t):=v^{T} C\left(I-\gamma^{-1} A\right)^{-1} \gamma^{-(t+1)} D(t)$ for $t \in \mathbb{N}_{0}$. An elementary calculation using (1.4) shows that

$$
z(\tau+1)-\gamma^{-1} A z(\tau) \in \gamma^{-1} B \gamma^{-\tau} F\left(\gamma^{\tau} C z(\tau)\right)+\gamma^{-(\tau+1)} D(\tau) \quad \forall \tau \in \mathbb{N}_{0} .
$$

Multiplying both sides of (3.11) by $v^{T} C\left(I-\gamma^{-1} A\right)^{-1}$ and invoking (3.10), we have that

$v^{T} C\left(I-\gamma^{-1} A\right)^{-1}(z(\tau+1)-z(\tau))+v^{T} C z(\tau) \in\left[0, \kappa v^{T} C z(\tau)\right]+H(\tau) \forall \tau \in \mathbb{N}_{0}$,

whence

$v^{T} C\left(I-\gamma^{-1} A\right)^{-1}(z(\tau+1)-z(\tau)) \in\left[-v^{T} C z(\tau),(\kappa-1) v^{T} C z(\tau)\right]+H(\tau) \quad \forall \tau \in \mathbb{N}_{0}$.

Defining $E(t):=\left[-v^{T} C z(t),(\kappa-1) v^{T} C z(t)\right]$ for $t \in \mathbb{N}_{0}$, it follows from (3.12) that there exist selections $\zeta$ and $\eta$ of $E$ and $H$, respectively, such that

$$
v^{T} C\left(I-\gamma^{-1} A\right)^{-1}(z(\tau+1)-z(\tau))=\zeta(\tau)+\eta(\tau) \quad \forall \tau \in \mathbb{N}_{0}
$$

Consequently,

$$
v^{T} C\left(I-\gamma^{-1} A\right)^{-1} z(t)=v^{T} C\left(I-\gamma^{-1} A\right)^{-1} x^{0}+\sum_{\tau=0}^{t-1}[\zeta(\tau)+h(\tau)] \quad \forall t \in \mathbb{N} .
$$

Noting that, by construction, for $\theta \in(0,1-\kappa)$

$$
\zeta(\tau)+\theta v^{T} C z(\tau) \leq 0, \quad \forall \tau \in \mathbb{N}_{0}
$$

we infer from (3.13) that

$$
\begin{aligned}
\theta \underset{v}{v}\|C z\|_{\ell^{1}(0, t-1)} & \leq \sum_{\tau=0}^{t-1} \theta v^{T} C z(\tau) \leq v^{T} C\left(I-\gamma^{-1} A\right)^{-1} z(t)+\sum_{\tau=0}^{t-1} \theta v^{T} C z(\tau) \\
& =v^{T} C\left(I-\gamma^{-1} A\right)^{-1} x^{0}+\sum_{\tau=0}^{t-1}\left[\zeta(\tau)+\theta v^{T} C z(\tau)+h(\tau)\right] \\
& \leq v^{T} C\left(I-\gamma^{-1} A\right)^{-1} x^{0}+\sum_{\tau=0}^{t-1} h(\tau) \quad \forall t \in \mathbb{N} .
\end{aligned}
$$

A straightforward estimate shows that there exists $K>0$ such that

$$
\sum_{\tau=0}^{t-1} h(\tau) \leq K \gamma^{-t} \delta(t) \quad \forall t \in \mathbb{N}
$$


where

$$
\delta(t):=\max _{\tau \in \underline{t-1}}\|D(\tau)\| \mid \forall t \in \mathbb{N} .
$$

In light of (3.14) and (3.15) we may infer the existence of $K_{1}, K_{2}>0$, independent of $x^{0}, t$ and $D$, such that

$$
\|C z\|_{\ell^{1}(0, t-1)} \leq K_{1}\left\|x^{0}\right\|+K_{2} \gamma^{-t} \max _{\tau \in \underline{t-1}}\|D(\tau)\| \quad \forall t \in \mathbb{N} .
$$

Invoking (3.11), $z$ satisfies the following inclusion

$$
\begin{aligned}
& z(t)-\left(\gamma^{-1} A\right)^{t} x^{0} \in \sum_{j=0}^{t-1}\left(\gamma^{-1} A\right)^{t-1-j} \\
& \times\left[\gamma^{-1} B \gamma^{-j} F\left(\gamma^{j} C z(j)\right)+\gamma^{-(j+1)} D(j)\right] \quad \forall t \in \mathbb{N},
\end{aligned}
$$

which, by (A3) and (3.9), may be estimated as follows:

$$
\|z(t)\| \leq K_{3}\left\|x^{0}\right\|+K_{4}\|C z\|_{\ell^{1}(0, t-1)}+K_{5} \gamma^{-t} \delta(t) \quad \forall t \in \mathbb{N},
$$

for positive constants $K_{3}, K_{4}$ and $K_{5}$, independent of $x^{0}, t$ and $D$. Inserting (3.16) into (3.17) establishes the claim with $\Gamma:=\max \left\{K_{3}+K_{1} K_{4}, K_{5}+K_{2} K_{4}\right\}$, as required.

Remark 3.2 In Sect. 2 we obtained two corollaries to Theorem 2.5-stability of certain non-autonomous differential inclusions and boundedness of solutions when the linear constraint (2.9) fails on a compact set, formulated as Corollary 2.9 and Corollary 2.12, respectively. The corresponding discrete-time versions of these results also hold mutatis mutandis, but, for the sake of brevity, are not formally stated.

\subsection{Lur'e difference equations and inequalities}

In parallel to Sect. 2.3, attention in the control theory literature has been devoted to the study of (forced) positive Lur'e difference equations of the form

$$
x(t+1)=A x(t)+B g(t, C x(t))+d(t), \quad x(0)=x^{0}, \quad t \in \mathbb{N}_{0},
$$

where $g: \mathbb{N}_{0} \times \mathbb{R}_{+}^{p} \rightarrow \mathbb{R}_{+}^{m}$ and $d: \mathbb{N}_{0} \rightarrow \mathbb{R}_{+}^{n}$. It is also of interest to study the stability properties of solutions of positive Lur'e difference inequalities, that is,

$$
0 \leq x(t+1)-A x(t) \leq B g(t, C x(t))+d(t), \quad x(0)=x^{0}, \quad t \in \mathbb{N}_{0},
$$

of which (3.18) is a special case. Both (3.18) and (3.19) are special cases of the forced positive Lur'e differential inclusion (1.4) with respective $F$ defined analogously to those in Sect. 2.3. 
The current section pertains to the difference inequalities (3.19) and we present a suite of discrete-time stability results which forms the discrete-time analogue of Corollary 2.13. The discrete-time version of the linear bound (2.54) is

$$
\|g(t, y)\| \leq R\|y\| \quad \forall t \in \mathbb{N}_{0}, \quad \forall y \in \mathbb{R}_{+}^{p},
$$

for some $R>0$.

Corollary 3.3 Given the forced positive Lur'e difference inequality (3.19), assume that (B1) and (B2) hold and that $g$ satisfies (3.20).

(i) If $d=0$ and there exists a strictly positive $v \in \mathbb{R}_{+}^{p}$ such that

$$
|\mathbf{G}(1) g(t, y)|_{v} \leq|y|_{v} \quad \forall y \in \mathbb{R}_{+}^{p}, \forall t \in \mathbb{N}_{0},
$$

then there exists $\Gamma>0$ such that, for all $x^{0} \in \mathbb{R}_{+}^{n}$, every solution $x$ of (2.53) satisfies

$$
\|x(t)\| \leq \Gamma\left\|x^{0}\right\| \quad \forall t \in \mathbb{N}_{0}
$$

(ii) If $d=0$ and there exist a strictly positive $v \in \mathbb{R}_{+}^{p}$ and a lower semi-continuous function $e: \mathbb{R}_{+}^{p} \rightarrow \mathbb{R}_{+}$such that

$$
e(y)>0 \text { and }|\mathbf{G}(1) g(t, y)|_{v}+e(y) \leq|y|_{v} \quad \forall y \in \mathbb{R}_{+}^{p} \backslash\{0\}, t \in \mathbb{N}_{0},
$$

then, for all $x^{0} \in \mathbb{R}_{+}^{n}$, every solution $x$ of (3.19) satisfies $x(t) \rightarrow 0$ as $t \rightarrow \infty$.

(iii) If there exist a strictly positive $v \in \mathbb{R}_{+}^{p}, \rho \in(0,1)$ and $\Theta \geq 0$ such that

$$
|\mathbf{G}(1) g(t, y)|_{v} \leq \rho|y|_{v} \quad \forall y \in \mathbb{R}_{+}^{p}, \quad\|y\| \geq \Theta, \forall t \in \mathbb{N}_{0},
$$

then there exist $\Gamma>0, \gamma \in(0,1)$ such that, for all $x^{0} \in \mathbb{R}_{+}^{n}$ and all $d \in$ $\ell_{\mathrm{loc}}^{\infty}\left(\mathbb{N}_{0} ; \mathbb{R}_{+}^{n}\right)$, every solution $x$ of (3.19) satisfies

$$
\|x(t)\| \leq \Gamma\left(\gamma^{t}\left\|x^{0}\right\|+\max _{\tau \in \underline{t-1}}\|d(\tau)\|+\beta\right) \quad \forall t \in \mathbb{N} .
$$

Here

$$
\beta=\beta(\Theta)=\|B\| \sup _{\|y\| \leq \Theta}\left(\sup _{t \in \mathbb{N}_{0}}(\operatorname{dist}(g(t, y), T(t, y)))\right),
$$

and

$$
T(t, y):=\left\{w \in[0, g(t, y)] \subseteq \mathbb{R}_{+}^{m}:|\mathbf{G}(1) w|_{v} \leq \rho|y|_{v}\right\}
$$

We conclude this section with some commentary on the discrete-time Lur'e system (3.18), and compare our results with others available in the literature. 
Remark 3.4 (a) Note that if $\Theta=0$ in statement (iii) of Corollary 3.3, then $\beta(\Theta)=0$ and the zero equilibrium pair of the Lur'e difference inequality (3.19) is exponentially ISS, that is, (3.3) holds. Consequently, if $\Theta=0$, then the zero equilibrium of the unforced $(d=0)$ Lur'e difference inequality is globally exponentially stable.

(b) The comments in Remark 2.14 pertaining to the continuous-time result Corollary 2.13 have discrete-time analogues in the context of Corollary 3.3. To avoid repetition, we do not repeat them here, other than noting that the statements (i)(iii) of Corollary 3.3 are similar to [59, Theorem 15], where different assumptions are made to those made here.

(c) Corollary 3.3 demonstrates that the weighted one-norm constraints imposed in (i)-(iii) are sufficient for respective notions of absolute stability. We claim that when $m=p=1$, the conclusions of statements (ii) and (iii) do not hold if their hypotheses are not satisfied. To that end, consider (3.18) with $m=p=1$, $d=0$ and a linear function $y \mapsto g(y)=\beta y$, for some $\beta>0$. Suppose further that $\beta$ and $\mathbf{G}(1)$ are such that (3.1) holds with equality (for any $0 \ll v^{T}$-when $p=1, v$ appears as a multiplicative scalar on both sides), that is,

$$
\mathbf{G}(1) \beta y=y \quad \forall y \geq 0 \text {. }
$$

Clearly, $\beta$ and $\mathbf{G}(1)$ must be related by

$$
\beta=\frac{1}{\mathbf{G}(1)}=\frac{1}{\|\mathbf{G}\|_{H^{\infty}}},
$$

and it is straightforward to prove that $r\left(A+\beta b c^{T}\right)=1$ meaning that $\beta$ is a destabilising perturbation. In particular, (3.18) becomes

$$
x(t+1)=\left(A+\beta b c^{T}\right) x(t), \quad x(0)=x^{0}, \quad t \in \mathbb{N}_{0},
$$

and so $\|x(t)\| \nrightarrow 0$ as $t \rightarrow \infty$ in general (dependent on $x^{0}$ ). We note that statement (i) of Corollary 3.3 holds, but that statements (ii) and (iii) do not. More generally, for arbitrary $g: \mathbb{R}_{+} \rightarrow \mathbb{R}_{+}$, whenever $y^{*}>0$ is such that

$$
\mathbf{G}(1) g\left(y^{*}\right)=y^{*}
$$

in particular meaning that the estimates in both (ii) and (iii) do not hold, then $x^{*}:=(I-A)^{-1} B g\left(y^{*}\right)$ is a non-zero equilibrium of (3.18) and the zero equilibrium of (3.18) cannot then be globally asymptotically or exponentially stable. The papers $[49,64]$ consider attractivity and stability properties of the non-zero equilibrium $x^{*}$ when $m=p=1$ and under conditions on $A, B, C$ and $g$ which ensure that $y^{*}>0$ in (3.21) is unique.

Remark 3.5 We provide some commentary comparing our results with material from the positive systems and control literature. The paper [63] (see also [65]) considers ISS for forced monotone dynamical systems, including those of the form 


$$
x(t+1)=\Gamma(x(t), v(t)), \quad x(0)=x^{0}, \quad t \in \mathbb{N}_{0},
$$

where $\Gamma: \mathbb{R}_{+}^{n} \times \mathbb{R}_{+}^{m} \rightarrow \mathbb{R}_{+}^{n}$ satisfies $\Gamma(0,0)=0$ and is monotone increasing, that is,

$$
\left(x_{1}, v_{1}\right) \leq\left(x_{2}, v_{2}\right) \Rightarrow \Gamma\left(x_{1}, v_{1}\right) \leq \Gamma\left(x_{2}, v_{2}\right) \quad \forall\left(x_{j}, v_{j}\right) \in \mathbb{R}_{+}^{n} \times \mathbb{R}_{+}^{m} .
$$

Clearly, in the case that the discrete-time Lur'e system (3.18) is autonomous, then it is an instance of (3.22) with $n=m$ and

$$
\mathbb{R}_{+}^{n} \times \mathbb{R}_{+}^{n} \ni(x, v) \mapsto \Gamma(x, v):=A x+B g(C x)+v \in \mathbb{R}_{+}^{n} .
$$

Moreover, if $g: \mathbb{R}_{+}^{p} \rightarrow \mathbb{R}_{+}^{m}$ is continuous and monotone with $g(0)=0$, then $\Gamma$ defined by (3.23) is continuous, monotone and satisfies $\Gamma(0,0)=0$. It is straightforward to prove that, under the assumptions of Corollary 3.3, if the first inequality in statement (iii) of Corollary 3.3 holds with $\Theta=0$, then $\Gamma$ given by (3.23) satisfies the inequality [63, Eq. (17)]. Hence, by [63, Theorem 3], it follows that the zero equilibrium pair of (3.18) is ISS. Similarly, in the unforced case, it can be shown that the first inequality in statement (ii) of Corollary 3.3 implies that

$$
s \in \mathbb{R}_{+}^{n} \text { and } \Gamma(s, 0) \geq s \Rightarrow s=0,
$$

and so global asymptotic stability of the zero equilibrium of (3.18) may be inferred from [62, Proposition 5.12]. Thus there is partial overlap between our results and those of $[62,63]$, in the special case that the Lur'e system under consideration is monotone. By way of contrast, we comment that the positive Lur'e systems considered throughout the current paper, although positive, need not be monotone.

\section{Examples}

We consider three worked examples.

Example 4.1 We consider stabilisation of single-input single-output (SISO) continuoustime, linear positive systems by quantised output feedback. Given

$$
\begin{aligned}
& \dot{x}=A x+b u+d, \quad x(0)=x^{0}, \\
& y=c^{T} x,
\end{aligned}
$$

where, as usual, $u, x, y$ and $d$ denote the input, state, output and exogenous disturbance, respectively, suppose that $A \in \mathbb{R}^{n \times n}$ is Metzler and $b, c \in \mathbb{R}_{+}^{n}$ are nonnegative, that $x^{0} \in \mathbb{R}_{+}^{n}$, and that $\alpha(A) \geq 0$, meaning that the uncontrolled linear system is unstable. The control objective is to stabilise (4.1) by static output feedback which is subject to quantisation. Specifically, for fixed $\tau>0$, consider the upper semi-continuous quantisation function $q: \mathbb{R}_{+} \rightarrow \mathbb{R}_{+}$defined by

$$
q(y):=\tau(m-1) \quad \forall y \in[(m-1) \tau, m \tau), \quad m \in \mathbb{N},
$$




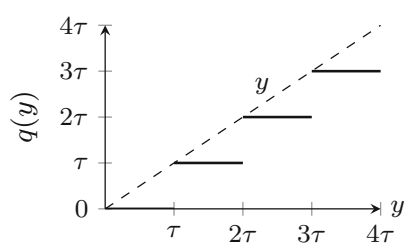

(a)

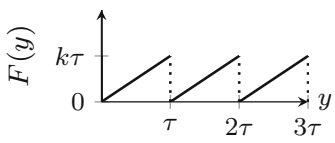

(b)

Fig. 2 a Quantisation function $q$ from (4.2) (solid line) and $y \mapsto y$ (dashed line). b Set-valued function $F$ with singleton-values in solid lines and interval-values in dotted lines

which is a coarse under-estimate of the identity function $y \mapsto y$, and an example of which is plotted in Fig. 2a.

We assume that $\mathbf{G} \neq 0$, where $\mathbf{G}$ is the transfer function of (4.1), and that there is a feedback-gain $k>0$ such that $A-k b c^{T}$ is Metzler and Hurwitz. With these assumptions we seek to apply Theorem 2.11 or Corollary 2.12 to investigate stability properties of the zero equilibrium of the linear system (4.1) in feedback connection with the quantised output feedback $u=-k q(y)$, together with the requirement that $x(t) \in \mathbb{R}_{+}^{n}$ for all $t \in \mathbb{R}_{+}^{n}$. The feedback connection may be formally written as

$$
\dot{x}=A x-k b q\left(c^{T} x\right)+d, \quad x(0)=x^{0} .
$$

Associated with $q$ and $k$ is the set-valued function $H_{k}: \mathbb{R}_{+} \rightarrow P_{0}\left(\mathbb{R}_{+}\right)$, given by

$$
H_{k}(y)=\left\{\begin{array}{ll}
k q(y) & y \in((m-1) \tau, m \tau) \\
{[(m-1) k \tau, m k \tau]} & y=m \tau
\end{array} \quad m \in \mathbb{N},\right.
$$

which "fills in the jumps" in $k q$ with closed intervals and leads to the differential inclusion for $x$

$$
\dot{x}-A x \in-b H_{k}\left(c^{T} x\right)+D, \quad x(0)=x^{0},
$$

where $D:=\{d\}$. To fit our current framework of positive Lur'e inclusions, we rewrite (4.3) as

$$
\dot{x}-\left(A-k b c^{T}\right) x \in b\left[k c^{T} x-H_{k}\left(c^{T} x\right)\right]+D(t), \quad x(0)=x^{0},
$$

which is in the form (1.3) with $A$ replaced by $A-k b c^{T}$ and $F: \mathbb{R}_{+} \rightarrow P_{0}\left(\mathbb{R}_{+}^{n}\right)$ given by $F(y):=k y-H_{k}(y)$, which is plotted in Fig. 2 b.

By our hypothesis on $k$ and construction of $H_{k}$, assumptions (A1)-(A3) are satisfied by the Lur'e inclusion (4.4). Further, $F$ has compact values and is upper semicontinuous (which is readily verified or see, for example [26, Example 1.3, p. 5]) and $D$ is locally bounded, measurable, with closed, convex values. Therefore, by Proposition 2.10, for every $x^{0} \in \mathbb{R}_{+}^{n}$, there is a global solution of (4.4) which satisfies $x(t) \in \mathbb{R}_{+}^{n}$ for all $t \in \mathbb{R}_{+}$. 
Let $\mathbf{G}_{k}$ denote the transfer function of the triple $\left(A-b k c^{T}, b, c^{T}\right)$, that is,

$$
\mathbf{G}_{k}=(I+k \mathbf{G})^{-1} \mathbf{G}
$$

We have that $\mathbf{G}_{k}(0)=\left\|\mathbf{G}_{k}\right\|_{H^{\infty}}$ is positive (indeed, we have that $\mathbf{G}=0$ if, and only if, $\mathbf{G}_{k}=0$ ).

For simplicity, assume that $\mathbf{G}$ is defined at zero. ${ }^{1}$ We consider two exhaustive cases. If $\mathbf{G}(0) \geq 0$, then $\mathbf{G}_{k}(0)=\mathbf{G}(0) /(1+k \mathbf{G}(0))=\rho / k$ for some $\rho \in[0,1)$, and noting from Fig. $2 b$ that

$$
w \leq k y \quad \forall w \in F(y), \forall y \geq 0,
$$

it follows that Theorem 2.11 applies and the zero equilibrium of (4.3) is exponentially ISS, which implies that $A$ is Hurwitz, which is false by hypothesis. We conclude that $\mathbf{G}(0)<0$, so that $\mathbf{G}_{k}(0)>1 / k$ and by choosing $\rho=\mathbf{G}_{k}(0)(k-\theta) \in(0,1)$ for $\theta$ sufficiently close to $k$, in light of Fig. $2 \mathrm{~b}$ it follows that

$$
\mathbf{G}_{k}(0) w \leq \rho y \quad \forall w \in F(y), \forall y \in \mathbb{R}_{+} y \geq \tau .
$$

Corollary 2.12 now applies, so that the zero equilibrium of (4.3) is exponentially ISS with bias, here with $\Theta$ in that result equal to $\tau$ and

$\beta=\|b\| \sup \left\{w-\rho y / \mathbf{G}_{k}(0): w \in F(y), 0 \leq y \leq \tau\right\}=\|b\|(k(1-\rho)-\rho / \mathbf{G}(0)) \tau$,

which, note, is a linear function of $\tau$.

For a numerical simulation, consider (4.1) with the following

$$
A=\left(\begin{array}{rrr}
-3 & 2 & 4 \\
2 & -1 & 5 \\
4 & 0 & -3
\end{array}\right), \quad B=\left(\begin{array}{l}
1 \\
0 \\
0
\end{array}\right), \quad C=\left(\begin{array}{lll}
0 & 1 & 1
\end{array}\right), \quad x^{0}=\left(\begin{array}{l}
10 \\
10 \\
10
\end{array}\right)
$$

Upon inspection, we see that $k=2$ renders $A-k B C=A-2 B C$ Metzler with $\alpha(A-2 B C)=-0.172<0$, so that $k=2$ is stabilising. Figure 3 plots solutions of (4.3) with $k=2$ for several $\tau>0$, subject to a nonnegative, periodic disturbance $d$ given by

$$
d(t)=0.3(1+\sin (0.6 t)) \quad t \in \mathbb{R}_{+} .
$$

The norm of the state decreases with decreasing $\tau$, corresponding to finer quantisation. For comparison, Fig. 3 also contains a plot of the solution of (4.1) subject to the output feedback $u=-k y$ (without quantisation).

We conclude by summarising that "linear positive stability", that is, $A-k b c^{T}$ Metzler and Hurwitz, is sufficient to ensure that the zero equilibrium pair of the closed-loop quantised feedback system is ISS with bias and the state remains positive. Consequently, the problem is one of static output feedback control design for linear positive systems via the choice of $k$, which has been considered in, for example [21].

\footnotetext{
1 Although this assumption is not needed, it eases exposition.
} 
Fig. 3 Numerical simulations of (4.3) with model data (4.5) and disturbance (4.6), $k=2$ and $\tau \in\{0.5,0.25,0.125\}$ in solid, dashed and dashed-dotted, respectively. The dotted line is un-quantised feedback system-the solution of (4.1) with $u=-k y$

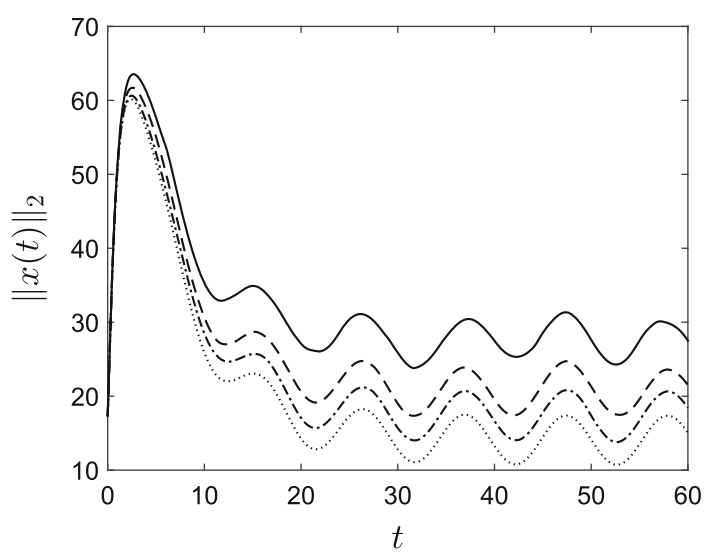

Observe that the constant $\Gamma$ depends only on $A, b, c, k$ and $\rho \in(0,1)$. Clearly, $\beta$ is a decreasing function of $\tau$, which captures the coarseness of the quantisation, and, as such, the ISS with bias bound for $\|x(t)\|$ decreases (viz. improves) with decreasing $\tau$, or finer quantisation, as we would expect, and as seen in Fig. 3.

Example 4.2 Consider the following model for a population partitioned into $n \in \mathbb{N}$, $n \geq 3$, discrete stage-classes:

$$
\left.\begin{array}{rl}
x_{1}(t+1)-s_{1} x_{1}(t) & \in F_{1}\left(\sum_{j=1}^{n} u_{1}(t) c_{j} x_{j}(t)\right) \sum_{j=1}^{n} u_{2}(t) c_{j} x_{j}(t), \quad x_{1}(0)=x_{1}^{0} \\
x_{2}(t+1)-s_{2} x_{2}(t) & \in F_{2}\left(x_{1}(t)\right) u_{3}(t) x_{1}(t), \quad x_{2}(0)=x_{2}^{0} \\
x_{i}(t+1) & =s_{i} x_{i}(t)+h_{i-1} x_{i-1}(t), \quad x_{i}(0)=x_{i}^{0}, \quad i \in\{3,4, \ldots, n\}
\end{array}\right\} t \in \mathbb{N}_{0} .
$$

Here $x_{i}(t)$ denotes the abundance or distribution of the $i$-th stage-class at time-step $t$. The $s_{i}$ and $h_{i}$ are probabilities denoting survival (or stasis) within a stage-class, and growth into the next stage-class, respectively. As such $s_{i}, h_{j} \in(0,1]$ and $s_{j}+h_{j} \leq 1$ for all $i \in\{1,2, \ldots, n\}$ and $j \in\{2, \ldots, n-1\}$. The $c_{i}$ terms are non-negative constants which capture the fecundity of the $i$-th stage-class. The set-valued functions $F_{1}$ and $F_{2}$ capture per-capita reproduction and per-capital survival rates, respectively, and are modelled thus to accommodate for uncertainty in accurately describing these processes. If $F_{1}$ and $F_{2}$ are constant singleton-valued functions, meaning that there is no density-dependence, then (4.7) may be expressed as matrix population projection model, see [70]. We are assuming, as is often the case, that recruitment is into the first stage-class, which typically denotes eggs, juveniles or seeds, in an insect, animal or plant model, respectively.

Finally, $u_{1}, u_{2}$ and $u_{3}$ are forcing (controls or disturbances) affecting the reproductive rates, the number of offspring of reproductive individuals and the number of individuals which transition from the first to second stage-classes, respectively. They are modelled to act multiplicatively so that, in particular, $u_{1}=u_{2}=u_{3} \equiv 1$ corresponds to the absence of forcing. In an animal model, to reduce a population, the term $u_{1}(t)<1$ could reflect applying contraceptives to (a proportion of) the population, 
for instance, and $u_{2}(t)<1$ could model the removal of new juvenile members of the population, whilst $u_{3}(t)<1$ corresponds to the removal of members of the population which are transitioning from first stage class into the second, perhaps by culling, harvesting or moving individuals.

For constant $u_{1}, u_{2}$ and $u_{3}$, the model (4.7) may be written in the form of (1.4) with

$$
\begin{aligned}
A & :=\left(\begin{array}{ccccc}
s_{1} & 0 & 0 & \ldots & 0 \\
0 & s_{2} & \ddots & \vdots & \vdots \\
\vdots & h_{2} & \ddots & \ddots & \\
\vdots & & \ddots & \ddots & 0 \\
0 & \ldots & 0 & h_{n-1} & s_{n}
\end{array}\right), \quad B:=\left(\begin{array}{cc}
1 & 0 \\
0 & 1 \\
\vdots & 0 \\
\vdots & \vdots \\
0 & 0
\end{array}\right), \quad C:=\left(\begin{array}{cccc}
c_{1} & c_{2} & \ldots & c_{n} \\
1 & 0 & \ldots & 0
\end{array}\right), \\
F\left(\left(\begin{array}{l}
y_{1} \\
y_{2}
\end{array}\right)\right) & :=\left(\begin{array}{l}
1 \\
0
\end{array}\right) F_{1}\left(u_{1} y_{1}\right) u_{2} y_{1}+\left(\begin{array}{l}
0 \\
1
\end{array}\right) F_{2}\left(y_{2}\right) u_{3} y_{2} .
\end{aligned}
$$

For ease of exposition in the present example, we address the following problem: assuming that

$$
F_{1}(y)=\left[f_{1}^{-}(y), f_{1}^{+}(y)\right] \text { and } F_{2}(y)=\left[f_{2}^{-}(y), f_{2}^{+}(y)\right] \quad \forall y \in \mathbb{R}_{+},
$$

for functions $f_{1}^{-}, f_{1}^{+}, f_{2}^{-}, f_{2}^{+}: \mathbb{R}_{+} \rightarrow \mathbb{R}_{+}$which satisfy

$$
f_{1}^{-}(y) \leq f_{1}^{+}(y) \text { and } f_{2}^{-}(y) \leq f_{2}^{+}(y) \quad \forall y \in \mathbb{R}_{+},
$$

find conditions on $\mathbf{G}(1), F_{1}, F_{2}, u_{1}, u_{2}$ and $u_{3}$ such that zero equilibrium of (4.7) is stable. The functions $f_{1}^{-}, f_{1}^{+}, f_{2}^{-}, f_{2}^{+}$are typically assumed to be continuous, non-negative valued and either sub-linear for large arguments or bounded. These latter qualitative properties seek to model density-dependent crowding, saturation and competition effects in the population, particularly at large abundances.

It is clear that $A, B$ and $C$ satisfy (B1) and as $\sigma(A)=\left\{s_{1}, \ldots, s_{n}\right\}$, it follows that $A$ satisfies (B2). An elementary calculation shows that

$$
\begin{aligned}
\mathbf{G}(1)= & C(I-A)^{-1} B=\left(\begin{array}{cccc}
c_{1} & c_{2} & \ldots & c_{n} \\
1 & 0 & \ldots & 0
\end{array}\right) \\
& \times\left(\begin{array}{ccccc}
1-s_{1} & 0 & 0 & \ldots & 0 \\
0 & 1-s_{2} & \ddots & \vdots & \vdots \\
\vdots & -h_{2} & \ddots & \ddots & \\
\vdots & & \ddots & \ddots & 0 \\
0 & \ldots & 0 & -h_{n-1} & 1-s_{n}
\end{array}\right)\left(\begin{array}{cc}
1 & 0 \\
0 & 1 \\
\vdots & 0 \\
\vdots & \vdots \\
0 & 0
\end{array}\right) \\
= & \left(\begin{array}{c|cc}
\frac{c_{1}}{1-s_{1}} \mid & \sum_{i=2}^{n} c_{i} \prod_{j=2}^{i} \frac{h_{j-1}}{1-s_{j}} \\
\hline \frac{1}{1-s_{1}} & 0
\end{array}\right.
\end{aligned}
$$


where $\alpha_{1}, \alpha_{2}, \alpha_{3} \geq 0$. To apply Theorem 3.1, we require $v^{T}=\left(v_{1} v_{2}\right) \gg 0$ and $\rho \in[0,1]$ such that

$$
v^{T} \mathbf{G}(1) w \leq \rho v^{T} y \quad \forall w \in F(y), \quad \forall y \in \mathbb{R}_{+}^{2},
$$

and, without loss of generality, we may assume that $v_{1}=1$. If $\rho=1$, then statement (i) of Theorem 3.1 applies and if $\rho<1$, then statement (iii) of Theorem 3.1 applies. Expanding the above inequality, we require $v_{2}>0$ such that

$$
\alpha_{1} w_{1}+\alpha_{2} w_{2}+v_{2} \alpha_{3} w_{1} \leq \rho y_{1}+\rho v_{2} y_{2} \quad \forall\left(\begin{array}{l}
w_{1} \\
w_{2}
\end{array}\right) \in F\left(\left(\begin{array}{l}
y_{1} \\
y_{2}
\end{array}\right)\right), \forall y_{1}, y_{2} \in \mathbb{R}_{+} .
$$

Using the definition of $F$, we see that (4.8) holds if

$$
\left(\alpha_{1}+v_{2} \alpha_{3}\right) f_{1}^{+}\left(u_{1} y_{1}\right) u_{2} y_{1} \leq \rho y_{1} \quad \text { and } \quad \alpha_{2} f_{2}^{+}\left(y_{2}\right) u_{3} y_{2} \leq \rho v_{2} y_{2} \quad \forall y_{1}, y_{2} \in \mathbb{R}_{+} \text {, }
$$

or, equivalently, if

$$
\left(\alpha_{1}+v_{2} \alpha_{3}\right) f_{1}^{+}\left(u_{1} y_{1}\right) u_{2} \leq \rho \quad \text { and } \quad \alpha_{2} f_{2}^{+}\left(y_{2}\right) u_{3} \leq \rho v_{2} \quad \forall y_{1}, y_{2} \in \mathbb{R}_{+} .
$$

It follows that (4.9) is a sufficient condition for stability of the zero equilibrium which is independent of the lower bounds $f_{1}^{-}$and $f_{2}^{-}$for $F$. Indeed, the "worst case" scenario from the point of view of destabilising the zero equilibrium corresponds to the upper bounds $f_{1}^{+}$and $f_{2}^{+}$for $F$, which both appear in (4.9). Furthermore, if $f_{1}^{+}$and $f_{2}^{+}$are assumed bounded, then (4.9) can be satisfied for $\rho<1$, if $u_{2}$ and $u_{3}$ are sufficiently small. Recall that in the present context control acts multiplicatively, and so "small" $u_{2}$ and $u_{3}$ correspond to "large" control efforts. For fixed $\alpha_{i}, f_{i}^{+}$and $\rho$, the first inequality in (4.9) may be satisfied by making $u_{2}$ sufficiently small. There is then some freedom in how small $u_{3}$ has to be to satisfy the second inequality, through the choice of $v_{2}$, which itself may be large. However, we note that the functions $z \mapsto f_{1}^{+}(z)$ and $z \mapsto f_{1}^{+}\left(u_{1} z\right)$ have the same image for each fixed positive $u_{1}$, and hence $u_{1}$ alone cannot necessarily guarantee that the first inequality in (4.9) is satisfied - and small $u_{2}$ may be required as well. Arguably these observations would have not been as apparent from the difference equations (4.7) alone.

Example 4.3 To illustrate the difference between Corollary 2.13 and the absolute stability results of [58], we consider (2.52), with $n=m=p=2$ and

$$
\mathbf{G}(0):=\left(\begin{array}{ll}
\alpha & \beta \\
\gamma & \delta
\end{array}\right), \quad g: \mathbb{R}_{+}^{2} \rightarrow \mathbb{R}_{+}^{2} \quad g(y):=\left(\begin{array}{l}
y_{2} \mathrm{e}^{-y_{1}} \\
y_{1} \mathrm{e}^{-y_{2}}
\end{array}\right) \quad \forall y=\left(\begin{array}{l}
y_{1} \\
y_{2}
\end{array}\right) \in \mathbb{R}_{+}^{2},
$$

where $\alpha, \beta, \gamma, \delta>0$ are parameters. Throughout this example we are considering the unforced version of (2.52), and so assume that $d=0$ in (2.52). A positive, stable realisation of $\mathbf{G}$ is

$$
A:=-I, \quad B:=I, \quad C:=\left(\begin{array}{ll}
\alpha & \beta \\
\gamma & \delta
\end{array}\right)
$$


which clearly satisfies assumptions (A1), (A2). It is readily seen that (A3) holds as well. We seek conditions on $\alpha, \beta, \gamma, \delta>0$ which ensure that there exists a strictly positive $v^{T}=\left(\begin{array}{ll}v_{1} & v_{2}\end{array}\right) \in \mathbb{R}_{+}^{2}$ such that

$$
v^{T} \mathbf{G}(0) g(y)<v^{T} y \quad \forall y=\left(\begin{array}{l}
y_{1} \\
y_{2}
\end{array}\right) \in \mathbb{R}_{+}^{2}, \quad y \neq 0,
$$

which, by statement (ii) of Corollary 2.13 guarantees that the zero equilibrium of (2.52) with model data (4.10) is globally asymptotically stable. Note that there is no loss of generality in assuming that $v_{1}=1$ and, expanding the above expression, we require that $\alpha, \beta, \gamma, \delta$ and $v_{2}$ satisfy

$$
\left(\alpha+v_{2} \gamma\right) y_{2} \mathrm{e}^{-y_{1}}+\left(\beta+v_{2} \delta\right) y_{1} \mathrm{e}^{-y_{2}}<y_{1}+v_{2} y_{2} \quad \forall y=\left(\begin{array}{l}
y_{1} \\
y_{2}
\end{array}\right) \in \mathbb{R}_{+}^{2}, \quad y \neq 0,
$$

which is certainly satisfied if

$$
\alpha+v_{2} \gamma \leq v_{2} \text { and } \beta+v_{2} \delta \leq 1
$$

In order to apply [58, Theorem 7.2] we require $M \in \mathbb{R}_{+}^{2 \times 2}$ such that

$$
g(y) \leq M y \quad \forall y \in \mathbb{R}_{+}^{2} \text { and } \mathbb{1}^{T} \mathbf{G}(0) M \ll \mathbb{1}^{T} .
$$

The first condition necessitates that

$$
N:=\left(\begin{array}{ll}
0 & 1 \\
1 & 0
\end{array}\right) \leq M
$$

and so substituting $M=N$ into the second condition yields

$$
\alpha+\gamma<1 \text { and } \beta+\delta<1
$$

which corresponds to taking $v_{2}=1$ in (4.11). Clearly, if $\beta+\delta \geq 1$, then (4.12) cannot hold, but if $\beta, \gamma<1$ and

$$
\frac{\alpha}{1-\gamma}<v_{2}<\frac{1-\beta}{\delta}
$$

then (4.11) holds, and so statement (ii) of Corollary 2.13 applies. Similar examples may be constructed in discrete-time.

\section{Discussion}

Global stability of the zero equilibrium of systems of positive Lur'e differential and difference inclusions has been considered. We have also considered input-to-state 
stability of the zero equilibrium pair of these inclusions when subject to exogenous forcing. Under rather mild assumptions on the set-valued term $F$, results available in the literature germane to the existence of local solutions of differential inclusions are readily applicable in the present context. Therefore, in the continuous-time case, we have focussed on stability of global solutions, and have presented conditions in terms of weighted one-norms of the "product" $\mathbf{G}(0) F(y)$ of the steady-state gain $\mathbf{G}(0)$ and set-valued nonlinearity $F$, reminiscent of classical small-gain conditions, which are sufficient for global stability properties, including ISS for forced inclusions. As we commented in Remark 2.6, sufficient conditions for the small-gain estimates are the existence of suitable nonnegative matrices $M$ such that linear constraints of the form $\mathbf{G}(0) w \leq M y$ for all $w \in F(y)$ and $y \in \mathbb{R}_{+}^{p}$ hold. The stability conditions presented guarantee existence of global solutions provided standard assumptions for existence of local solutions are satisfied. Our main results are Theorems 2.5 and 2.11 in the continuous-time case, and Theorem 3.1 in discrete-time. As corollaries we obtained a so-called ISS with bias result, Corollary 2.12, for forced inclusions where the small-gain condition fails on some bounded set, and suites of stability results for systems of positive Lur'e differential and difference equations and inequalities, Corollaries 2.13 and 3.3. Two key differences to our earlier work, notably [60], is that the linear components of the Lur'e inclusions are multivariable and, second, here we consider differential inclusions, rather than differential equations. Comparisons to other relevant results available in the literature appear in Remarks 2.14, 3.4 and 3.5 and worked Examples were presented in Sect. 4.

Acknowledgements B. Rüffer has been supported by ARC grant DP160102138.

Open Access This article is distributed under the terms of the Creative Commons Attribution 4.0 International License (http://creativecommons.org/licenses/by/4.0/), which permits unrestricted use, distribution, and reproduction in any medium, provided you give appropriate credit to the original author(s) and the source, provide a link to the Creative Commons license, and indicate if changes were made.

\section{References}

1. Haddad, W.M., Chellaboina, V., Hui, Q.: Nonnegative and Compartmental Dynamical Systems. Princeton University Press, Princeton (2010)

2. Krause, U.: Positive Dynamical Systems in Discrete Time. De Gruyter, Berlin (2015)

3. Berman, A., Neumann, M., Stern, R.J.: Nonnegative Matrices in Dynamic Systems. Wiley, New York (1989)

4. Krasnosel'skij, M.A., Lifshits, J.A., Sobolev, A.V.: Positive Linear Systems: The Method of Positive Operators. Heldermann Verlag, Berlin (1989)

5. Berman, A., Plemmons, R.J.: Nonnegative Matrices in the Mathematical Sciences. SIAM, Philadelphia (1994)

6. Luenberger, D.G.: Introduction to Dynamic Systems: Theory, Models, and Applications. Wiley, New York (1979)

7. Gaubert, S., Gunawardena, J.: The Perron-Frobenius theorem for homogeneous, monotone functions. Trans. Am. Math. Soc. 356, 4931-4950 (2004)

8. Sine, R.: A nonlinear Perron-Frobenius theorem. Proc. Am. Math. Soc. 109, 331-336 (1990)

9. Lemmens, B., Nussbaum, R.: Nonlinear Perron-Frobenius Theory. Cambridge University Press, Cambridge (2012)

10. Smith, H.L.: Monotone Dynamical Systems. American Mathematical Society, Providence (1995) 
11. Hirsch, M.W., Smith, H.: Monotone dynamical systems. In: Cañada, A., Drábek, P., Fonda, A. (eds.) Handbook of Differential Equations: Ordinary Differential Equations, vol. II, pp. 239-357. Elsevier B.V., Amsterdam (2005)

12. Hirsch, M.W., Smith, H.L.: Monotone maps: a review. J. Difference Equ. Appl. 11, 379-398 (2005)

13. Farina, L., Rinaldi, S.: Positive Linear Systems: Theory and Applications. Wiley, New York (2000)

14. Rantzer, A.: Scalable control of positive systems. Eur. J. Control 24, 72-80 (2015)

15. Coxson, P.G., Shapiro, H.: Positive input reachability and controllability of positive systems. Linear Algebra Appl. 94, 35-53 (1987)

16. Caccetta, L., Rumchev, V.G.: A survey of reachability and controllability for positive linear systems. Ann. Oper. Res. 98, 101-122 (2001)

17. Härdin, H.M., van Schuppen, J.H.: Observers for linear positive systems. Linear Algebra Appl. 425, 571-607 (2007)

18. Ohta, Y., Maeda, H., Kodama, S.: Reachability, observability, and realizability of continuous-time positive systems. SIAM J. Control Optim. 22, 171-180 (1984)

19. Benvenuti, L., Farina, L.: A tutorial on the positive realization problem. IEEE Trans. Automat. Control 49, 651-664 (2004)

20. De Leenheer, P., Aeyels, D.: Stabilization of positive linear systems. Syst. Control Lett. 44, 259-271 (2001)

21. Roszak, B., Davison, E.J.: Necessary and sufficient conditions for stabilizability of positive LTI systems. Syst. Control Lett. 58, 474-481 (2009)

22. Hinrichsen, D., Pritchard, A.J.: Stability radii of linear systems. Syst. Control Lett. 7, 1-10 (1986)

23. Hinrichsen, D., Pritchard, A.J.: Stability radius for structured perturbations and the algebraic Riccati equation. Syst. Control Lett. 8, 105-113 (1986)

24. Son, N.K., Hinrichsen, D.: Robust stability of positive continuous time systems. Numer. Funct. Anal. Optim. 17, 649-659 (1996)

25. Aubin, J.-P., Cellina, A.: Differential Inclusions. Springer, Berlin (1984)

26. Deimling, K.: Multivalued Differential Equations. Walter de Gruyter \& Co., Berlin (1992)

27. Vrabie, I.I.: Differential Equations. World Scientific Publishing Co., Inc., River Edge (2004)

28. Liberzon, D.: Calculus of Variations and Optimal Control Theory. Princeton University Press, Princeton (2012)

29. Vinter, R.: Optimal Control. Birkhäuser, Boston (2000)

30. Clarke, F.H.: Optimization and Nonsmooth Analysis, 2nd edn. SIAM, Philadelphia (1990)

31. Aubin, J.-P., Frankowska, H.: Set-Valued Analysis. Birkhäuser, Boston (1990)

32. Goebel, R., Sanfelice, R.G., Teel, A.R.: Hybrid dynamical systems: robust stability and control for systems that combine continuous-time and discrete-time dynamics. IEEE Control Syst. Mag. 29, 28-93 (2009)

33. Ryan, E.P.: Discontinuous feedback and universal adaptive stabilization. In: Hinrichsen, D., Mårtensson, B. (eds.) Control of Uncertain Systems, pp. 245-258. Birkhäuser, Boston (1990)

34. Ryan, E.P.: On Brockett's condition for smooth stabilizability and its necessity in a context of nonsmooth feedback. SIAM J. Control Optim. 32, 1597-1604 (1994)

35. Cortés, J.: Discontinuous dynamical systems: a tutorial on solutions, nonsmooth analysis, and stability. IEEE Control Syst. Mag. 28, 36-73 (2008)

36. Jayawardhana, B., Logemann, H., Ryan, E.: The circle criterion and input-to-state stability. IEEE Control Syst. Mag. 31, 32-67 (2011)

37. De Glas, M.: Exponential stability revisited. Int. J. Control 46, 1505-1510 (1987)

38. Liberzon, M.: Essays on the absolute stability theory. Autom. Remote Control 67, 1610-1644 (2006)

39. Vidyasagar, M.: Nonlinear Systems Analysis. SIAM, Philadelphia (2002)

40. Khalil, H.K.: Nonlinear Systems. Macmillan Publishing Company, New York (1992)

41. Haddad, W.M., Chellaboina, V.: Nonlinear Dynamical Systems and Control. Princeton University Press, Princeton (2008)

42. Bill, A., Guiver, C., Logemann, H., Townley, S.: The converging-input converging-state property for Lur'e systems. Math. Control Signals Syst. 29, 1-50 (2017)

43. Briat, C.: Robust stability and stabilization of uncertain linear positive systems via integral linear constraints: $L_{1}$-gain and $L_{\infty}$-gain characterization. Int. J. Robust Nonlinear Control 23, 1932-1954 (2013)

44. Rantzer, A., Rüffer, B., Dirr, G.: Separable Lyapunov functions for monotone systems. In: 52nd IEEE Conference on Decision and Control, Florence (2013) 
45. Sontag, E.D.: Smooth stabilization implies coprime factorization. IEEE Trans. Automat. Control 34, 435-443 (1989)

46. Sontag, E.D.: Input to State Stability: Basic Concepts and Results, in Nonlinear and Optimal Control Theory, pp. 163-220. Springer, Berlin (2008)

47. Sarkans, E., Logemann, H.: Input-to-state stability of Lur'e systems. Math. Control Signals Syst. 27, 439-465 (2015)

48. Jayawardhana, B., Logemann, H., Ryan, E.P.: Input-to-state stability of differential inclusions with applications to hysteretic and quantized feedback systems. SIAM J. Control Optim. 48, 1031-1054 (2009)

49. Townley, S., Rebarber, R., Tenhumberg, B.: Feedback control systems analysis of density dependent population dynamics. Syst. Control Lett. 61, 309-315 (2012)

50. Rebarber, R., Tenhumberg, B., Townley, S.: Global asymptotic stability of density dependent integral population projection models. Theor. Popul. Biol. 81, 81-87 (2012)

51. Smith, H.L., Thieme, H.R.: Persistence and global stability for a class of discrete time structured population models. Discrete Contin. Dyn. Syst. 33, 4627-4646 (2013)

52. Franco, D., Logemann, H., Perán, J.: Global stability of an age-structured population model. Syst. Control Lett. 65, 30-36 (2014)

53. Eager, E.A., Rebarber, R., Tenhumberg, B.: Global asymptotic stability of plant-seed bank models. J. Math. Biol. 69, 1-37 (2014)

54. Taylor, C.M., Hastings, A.: Allee effects in biological invasions. Ecol. Lett. 8, 895-908 (2005)

55. Courchamp, F., Berec, L., Gascoigne, J.: Allee Effects in Ecology and Conservation. Oxford University Press, Oxford (2008)

56. Schreiber, S.J.: Allee effects, extinctions, and chaotic transients in simple population models. Theor. Popul. Biol. 64, 201-209 (2003)

57. Eager, E., Rebarber, R., Tenhumberg, B.: Choice of density-dependent seedling recruitment function affects predicted transient dynamics: a case study with platte thistle. Theor. Ecol. 5, 1-15 (2011)

58. Haddad, W.M., Chellaboina, V.: Stability and dissipativity theory for nonnegative dynamical systems: a unified analysis framework for biological and physiological systems. Nonlinear Anal. Real World Appl. 6, 35-65 (2005)

59. Haddad, W.M., Chellaboina, V.S., August, E.: Stability and dissipativity theory for discrete-time nonnegative and compartmental dynamical systems. Int. J. Control 76, 1845-1861 (2003)

60. Bill, A., Guiver, C., Logemann, H., Townley, S.: Stability of nonnegative Lur'e systems. SIAM J. Control Optim. 54, 1176-1211 (2016)

61. Krause, U., Ranft, P.: A limit set trichotomy for monotone nonlinear dynamical systems. Nonlinear Anal. 19, 375-392 (1992)

62. Dashkovskiy, S., Rüffer, B.S., Wirth, F.R.: Discrete time monotone systems: criteria for global asymptotic stability and applications. In: Proceeding of 17th International Symposium on Mathematical Theory of Networks and Systems (Kyoto, Japan), pp. 89-97 (2006)

63. Rüffer, B.S., Sailer, R.: Input-to-state stability for discrete-time monotone systems. In: Proceeding of 21st International Symposium Mathematical Theory of Networks and Systems (MTNS), pp. 96-102 (2014)

64. Franco, D., Guiver, C., Logemann, H., Peràn, J.: Semi-global persistence and stability for a class of forced discrete-time population models. Physica D 360, 46-61 (2017)

65. Rüffer, B.S.: Small-gain conditions and the comparison principle. IEEE Trans. Automat. Control 55, 1732-1736 (2010)

66. Plemmons, R.J.: $M$-matrix characterizations. I. Nonsingular $M$-matrices. Linear Algebra Appl. 18, 175-188 (1977)

67. Yakubovich, V.A., Leonov, G.A., Gelig, A.K.: Stability of Stationary Sets in Control Systems with Discontinuous Nonlinearities. World Scientific Publishing Co., Inc., River Edge (2004)

68. Jiang, Z.P., Teel, A.R., Praly, L.: Small-gain theorem for ISS systems and applications. Math. Control Signals Syst. 7, 95-120 (1994)

69. Sontag, E.D., Wang, Y.: New characterizations of input-to-state stability. IEEE Trans. Automat. Control 41, 1283-1294 (1996)

70. Caswell, H.: Matrix Population Models: Construction, Analysis, and Interpretation. Sinauer Associates, Sunderland (2001) 